\title{
Possibilistic Randomisation in Strategic-form Games
}

\author{
Hykel Hosni \\ Dipartimento di Filosofia, Università degli Studi di Milano, Italy \\ Enrico Marchioni \\ School of Electronics and Computer Science, University of Southampton, UK
}

\begin{abstract}
Since the seminal work of John Nash, convex combinations of actions are known to guarantee the existence of equilibria in strategic-form games. This paper introduces an alternative notion of randomisation among actions - possibilistic randomisation - and investigates the mathematical consequences of doing so. The framework of possibility theory gives rise to two distinct notions of equilibria both of which are characterised in our main results: a qualitative one based on the Sugeno integral and a quantitative one based on the Choquet integral. Then the two notions of equilibrium are compared against a coordination game with payoff-distinguishable equilibria known as the Weak-link game.
\end{abstract}

Keywords: Possibilistic Radomisation, Mixed Strategies, Possibilistic Expected Utility, Nash Equilibria, Weak-link Game, Selection of Multiple Equilibria

\section{Introduction and motivation}

A key foundational question in game theory is to do with defining solution concepts for strategic situations in which players interact rationally. To accomplish this normative task, rationality is defined as the maximisation of the player's own welfare (as they perceive it) subject to the predictions made by their opponents, all of whom are assumed to be rational. Moreover it is assumed that players have common knowledge of each other's rationality. In the standard analysis of strategic-form games, (also known as static games or simultaneous-moves games), the individual rationality of players is defined as the maximisation of complete and transitive preferences over the payoffs of the game, i.e. the consequences of their actions. A central result is due to John Nash who elaborates on work by Cournot, Borel and von Neumann, among others (see [41] for a terse historical account). Nash defined a profile of actions/strategies (one for each player) an equilibrium if each player's action maximises their expected payoff given the other players' actions. Nash's seminal 1950 result identifies the rather general conditions under which such an equilibrium is guaranteed to exist.

In the particular context of two-person zero-sum games, von Neumann had observed in 1928 that not all games admit solutions in what would be later called pure actions. As noted, among others by Luce and Raiffa in their classic [35] (p.70) this opens up two scenarios. In the first, players give up playing, because no profile of strategies satisfies the conditions for being an equilibrium. This scenario, though, is not compatible with the normative goal of the theory. Hence the only viable alternative is to interpret the indecisiveness which arises from the lack of equilibria in pure 
actions as indifference among which action should be selected as one's own half of an equilibrium point. In the decision-theoretic tradition such an indifference has been formalised by assuming that each player delegates their choice of action to a randomising device, the obvious choice being a probability distribution over the set of actions, called a mixed strategy.

So, the idea of mixing actions or strategies entered game theory pretty much as $i$ entered algebra: as a mathematical device sufficient to provide a solution to all problems of a certain kind. Just like extending $\mathbb{R}$ to the complex field makes all quadratic equations solvable, similarly allowing for mixed actions guarantees that all games have a Nash equilibrium.

Let us pause for a second and ask why the randomising device which has been used to define mixed strategies turned out probability distributions. The reasons for this choice are all too clear: during the 1920s probability was well understood in connection to randomness and possibly the only mathematical way of representing it. In addition, randomising pure strategies through probability distributions gives rise to convex sets from which to make choices, and this pays significant and well-known mathematical dividends.

It wasn't until the late 1960's that game theorists took a closer look at the meaning of mixed strategies, and sought to give it a behavioural foundation. This was achieved in [30], where J. Harsanyi identified the conditions under which equilibria in mixed strategies in a game of perfect information can be seen as equilibria in pure actions of a corresponding game of imperfect information. This method, known as purification, provides a behavioural foundation - i.e. a tangible game theoretic meaning - to the notion of mixed strategy by turning an equilibrium on random acts into an equilibrium in beliefs (about player's types). Hence there is an intriguing and subtle relation between the notion of randomisation in games and the behavioural interpretation of it.

\subsection{The goal and structure of this paper}

Our concern in this paper is with the mathematical, as opposed to behavioural, interpretation of mixed strategies. Much work has been done in the past few decades on non-probabilistic measures of uncertainty (see $[29,47]$ ) and this clearly allows for alternative notions of randomisation. It is only natural then that we take one big step back and model indifference among actions by means of non-probabilistic randomisation. The goal of this paper is to investigate the mathematical consequences of assuming that randomisations of actions in strategic-form games games occur by attaching to each set of pure actions a possibility distribution. We defer to future work the related, but quite distinct, question of investigating possibilistic equilibria in beliefs.

Possibility theory [18] has been developed over the past few decades as a model of uncertainty capable of representing incomplete information, rather than uncertainty quantification. Informally speaking possibility theory allows for a more coarse-grained (and more human-like [45]) representation of uncertainty than probability theory, accommodating both qualitative and quantitative approaches [20]. This twofold nature made possibility theory relevant in a variety of domains, from logic-based artificial intelligence [21], to decision theory [19, 15, 22] and statistics [13]. This work aims at making the first steps toward bringing the key concepts of possibility theory to bear the foundations of (non-cooperative) game theory.

The paper is organised as follows. Section 2 provides the relevant background on strategicform games and possibility theory. In particular, we introduce the notions of Choquet and Sugeno integrals for possibility measures that underpin two alternative concepts of expected utility in possibility theory. The main contribution of the paper is presented in Section 3. We begin by expanding strategic-form games with possibilistic mixed strategies, which are possibility distributions over a player's strategy set, and define the concepts of Choquet and Sugeno equilibrium. 
Possibilistic mixed strategies are not given here any specific interpretation, and are simply taken as an alternative way to represent the act of randomisation. Our main results, Theorem 13 and Theorem 22, identify the exact conditions a profile of possibilistic mixed strategies must satisfy to be a Choquet and Sugeno equilibrium, respectively.

Since possibilistic randomisation gives rise to two distinct notions of equilibrium, it is natural to articulate a comparison between them. We do so through the analysis of a weak-link game (a.k.a minimum effort game) in Section 4. The reason we regard this game as one of particular interest for the purposes of this paper lies in the fact that it is a coordination game, i.e. one in which each combination of the same action yields an equilibrium (in pure strategies). Whilst those equilibria can be distinguished by their payoffs, a player who selects their part of a Nash equilibrium cannot make this distinction. Hence Nash equilibrium cannot guide players to coordinate on the payoffefficient action profiles. With Theorem 27 we give a characterisation, specific for weak-link games, of when a pair of possibilistic mixed strategies forms a Choquet equilibrium. Here, by explicitly interpreting possibilistic mixed strategies as an index of commitment, we show that Theorem 27 leads to the following interpretation: for a weak-link game, a pair of possibilistic mixed strategies forms a Choquet equilibrium provided that each disagreement in the level of commitment that each player has with respect to a given strategy pair is compensated by an agreement on a strategy pair with a higher payoff. We argue that this approach shows that, when applied to weak-link games, the concept of a Choquet equilibrium offers a refinement of Nash equilibria.

We end this article with some final considerations on the content and scope of this paper and on future work.

\subsection{Related literature}

Not much work has been devoted to explore the relevance of possibility theory to game theory, as we pointed out in our preliminary investigations on the subject $[32,33,34]$. In those papers we introduced the notions of a possibilistic game and possibilistic mixed strategy, along with a concept of possibilistic equilibrium based on the Sugeno integral [48] (called in the present work "Sugeno equilibrium"). In [34], we showed that equilibria of this kind always exist and characterised the conditions for a possibilistic mixed strategy combination to be an equilibrium (characterisation which we improve in the present article). The work by Ben Amor, Fargier and Sabbadin [1] later introduced a notion of strategic-form games with possibilistic mixed strategies and possibilistic equilibria corresponding to those previously defined in [34]. Not directly related to our work is the paper by De Clercq et al. [8], who study the use of possibilistic logic [21] to model situations in which agents are uncertain about other agents' goals in Boolean games [25], i.e. logic-based games where players' utilities are represented by Boolean formulas.

In a recent article, Radul [44] investigates with topological techniques the concept of equilibrium proposed in [23] for games where players' beliefs about their opponents' behaviour are modelled as possibility measures and expectation is computed with the Sugeno integral. This kind of equilibria is shown to always exist, but note the shift in the main question, which is no longer on how to randomise in non-probabilistic ways but is how to generalise equilibria in beliefs beyond probability.

\section{Background}

\subsection{Strategic-form Games}

For present purposes it is sufficient to recall a selection of key definitions for static, non-cooperative, games in strategic form. All the games in this article are assumed to be two-person games. In- 
terested readers can refer to classic introductions such as [43], the more recent [39], or the freely available [2].

Definition 1 (Strategic-form Game). A strategic-form game is a tuple

$$
\mathbf{G}=\left\langle N, S_{1}, S_{2}, u_{1}, u_{2}\right\rangle
$$

where:

1. $N=\{1,2\}$ is the set of players of the game.

2. $S_{i}$ is a finite set of strategies for each player $i \in\{1,2\}$, also called strategy space.

3. $u_{i}: S_{1} \times S_{2} \rightarrow \mathbb{R}^{+}$, for each player $i \in\{1,2\}$, is a non-negative real-valued function (different from the identically zero function) called utility function (or payoff function).

Given a player $i \in\{1,2\}$, we sometimes refer to the other player as $-i$. The elements of each $S_{i}$ are often referred to as pure strategies. We usually denote by $s_{i}$ an arbitrary strategy for player $i$. Given $s_{i} \in S_{i}, s_{-i} \in S_{-i}$ is used to denote a strategy for the other player. A strategy combination is any pair $\left(s_{1}, s_{2}\right) \in S_{1} \times S_{2}$.

Definition 2 (Best response). Let $\mathbf{G}$ be a strategic-form game. Player's 1 best response to any $s_{2} \in S_{2}$ is a strategy $s_{1} \in S_{1}$ such that for all $s_{1}^{\prime} \in S_{1}$

$$
u_{i}\left(s_{1}, s_{2}\right) \geq u_{i}\left(s_{1}^{\prime}, s_{2}\right) .
$$

The definition for Player 2 is analogous.

Definition 3 (Pure Strategy Nash Equilibrium). Let $\mathbf{G}$ be a strategic-form game. We call a pair of pure strategies $\left(s_{1}, s_{2}\right) \in S_{1} \times S_{2}$ a Nash equilibrium (NE, for short) if each player's strategy is a best response to the other player's strategy.

It is well known that not all strategic-form games admit a pure strategy Nash equilibrium. This situation is obviated by allowing players to not simply choose one among their pure strategies, but among all possible mixed strategies, i.e. all probability distributions over their strategy set. More formally:

Definition 4 (Mixed Strategy). In a strategic-form game $\mathbf{G}$, a mixed strategy $\sigma_{i}$ for player $i$ is a probability distribution over the set of strategies $S_{i}$, i.e. a function $\sigma_{i}: S_{i} \rightarrow[0,1]$ such that

$$
\sum_{s_{i} \in S_{i}} \sigma_{i}\left(s_{i}\right)=1 .
$$

Similar to pure strategies, any pair of mixed strategies $\left(\sigma_{1}, \sigma_{2}\right)$ is called a mixed strategy profile or mixed strategy combination.

Definition 5 (Mixed Extension). Let

$$
\mathbf{G}=\left\langle N, S_{1}, S_{2}, u_{1}, u_{2}\right\rangle
$$

be a strategic-form game. The mixed extension of $\mathbf{G}$ is the game

$$
\mathfrak{G}=\left\langle N, M S_{1}, M S_{2}, e u_{1}, e u_{2}\right\rangle
$$

where, for $i \in\{1,2\}$ : 
- Each $M S_{i}$ is the set of all mixed strategies of player $i$ over $S_{i}$.

- Each eu $u_{i}: M S_{1} \times M S_{2} \rightarrow \mathbb{R}$ is a payoff function that associates with each mixed strategy combination $\left(\sigma_{1}, \sigma_{2}\right)$ its expected utility

$$
e u_{i}\left(\sigma_{1}, \sigma_{2}\right)=\sum_{\left(s_{1}, s_{2}\right) \in S_{1} \times S_{2}}\left(\left(\sigma_{1}\left(s_{1}\right) \cdot \sigma_{2}\left(s_{2}\right)\right) \cdot u_{i}\left(s_{1}, s_{2}\right)\right) .
$$

Both the concept of best response and Nash equilibrium in pure strategies are easily generalised to mixed strategies.

Definition 6 (Best Response: Mixed Strategies). Let $\mathbf{G}$ a strategic-form game and let $\mathfrak{G}$ be its mixed extension. Player 1 's mixed strategy $\sigma_{1}$ is called a best response to $\sigma_{2}$ if, for all $\sigma_{1}^{\prime} \in M S_{1}$,

$$
e u_{1}\left(\sigma_{1}, \sigma_{2}\right) \geq e u_{1}\left(\sigma_{1}^{\prime}, \sigma_{2}\right) .
$$

The definition for Player 2 is analogous.

Definition 7 (Mixed Strategy Nash Equilibrium). Let $\mathbf{G}$ a strategic-form game and let $\mathfrak{G}$ be its mixed extension. We call a pair of mixed strategies $\left(\sigma_{1}, \sigma_{2}\right) \in M S_{1} \times M S_{2}$ a mixed strategy Nash equilibrium if each player's mixed strategy is a best response to the other player's mixed strategy.

The following celebrated theorem by John Nash shows that every strategic-form game admits an equilibrium in mixed strategies.

Theorem 8 (Nash, 1951). Every strategic-form game has mixed extension that admits mixed strategy Nash equilibria.

\subsection{Possibility Measures, Choquet and Sugeno Integration}

In this section, we provide the background notions concerning possibility measures and distributions, and Choquet and Sugeno integration. The reader can find a full account of these topics in $[9,10,11,20,27]$. In what follows, we will use the symbols $\wedge$ and $\vee$ to represent the functions corresponding to the minimum and maximum of real numbers, respectively.

Given a set $X$, a set function is a mapping $\mu: 2^{X} \rightarrow \mathbb{R}$ assigning a real number to each set in $2^{X}$. A set function $\mu$ is called:

1. Grounded, if $\mu(\emptyset)=0$;

2. Normalised, whenever $\mu(X)=1$;

3. Monotone, if, for all $A, B \in 2^{X}$ such that $A \subseteq B, \mu(A) \leq \mu(B)$;

4. Maxitive, if, for all $A, B \in 2^{X}, \mu(A \cup B)=\mu(A) \vee \mu(B)$.

A capacity is a grounded monotone set function.

A possibility measure $\Pi: 2^{X} \rightarrow[0,1]$ is a normalised and maxitive capacity. A possibility distribution is a function $\pi: X \rightarrow[0,1]$ such that

$$
\bigvee_{x \in X} \pi(x)=1
$$


Given a possibility distribution $\pi$ on a set $X$, the function $\Pi: 2^{X} \rightarrow[0,1]$ such that for all $A \in 2^{X}$

$$
\Pi(A)=\sup _{x \in A} \pi(x)
$$

is a possibility measure called the possibility measure generated from $\pi$. Given a possibility measure $\Pi: 2^{X} \rightarrow[0,1]$, the function $\pi: X \rightarrow[0,1]$ defined $\pi(x)=\Pi(\{x\})$ is a possibility distribution.

We call a non-empty subcollection $\mathcal{F}$ of $2^{X}$ an algebra, whenever $\mathcal{F}$ is closed under finite union and complementation. Given a set $X$ and an algebra $\mathcal{F}$, a function $f: X \rightarrow \mathbb{R}$ is called $\mathcal{F}$-measurable if the sets

$$
\{x: f(x)>t\} \quad \text { and } \quad\{x: f(x) \geq t\}
$$

belong to $\mathcal{F}$ for all $t \in \mathbb{R}$.

Let $f$ be a bounded non-negative $\mathcal{F}$-measurable function and let $\Pi$ be a possibility measure. The Choquet integral of $f$ with respect to $\Pi$ is defined as

$$
\int^{\mathcal{C h}} f \mathrm{~d} \Pi=\int_{0}^{\infty} \Pi(\{x \in X: f(x) \geq t\}) \mathrm{d} t
$$

with $t \in \mathbb{R}$, where the right-hand side is the Riemann integral (see [7]).

As usual with Sugeno integration, the function $f$ is assumed to have the same range as the possibility measure (see $[6,9,10]$ ). This range is usually assumed to be $[0,1]$, but, in both cases, it can be any interval $[a, b] \subset \mathbb{R}$. So, let $f: X \rightarrow[0,1]$ be a $\mathcal{F}$-measurable function and let $\Pi$ be a possibility measure. The Sugeno integral of $f$ with respect to $\Pi$ is defined as

$$
\int^{\mathcal{S}} f \mathrm{~d} \Pi=\bigvee_{t \in[0,1]}(\Pi(\{x \in X: f(x) \geq t\}) \wedge t)
$$

(see [48]).

Suppose that $X=\left\{x_{1}, \ldots, x_{n}\right\}$ is a finite set and that $\mathcal{F}=2^{X}$. Every function $f: X \rightarrow \mathbb{R}$ is clearly $\mathcal{F}$-measurable and is called a finite function. Both the Choquet and the Sugeno integral of a finite function take a simpler form. Take a permutation $\alpha$ over $X$ such that

$$
f\left(x_{\alpha(1)}\right) \leq f\left(x_{\alpha(2)}\right) \leq \ldots \leq f\left(x_{\alpha(n)}\right),
$$

and let

$$
A_{\alpha(j)}=\left\{x_{\alpha(j)}, \ldots, x_{\alpha(n)}\right\} .
$$

The Choquet integral and the Sugeno integral of a finite function $f$ with respect to a possibility measure $\Pi$ have the following form:

$$
\int^{\mathcal{C} h} f \mathrm{~d} \Pi=\sum_{j=1}^{n}\left(f\left(x_{\alpha(j)}\right)-f\left(x_{\alpha(j-1)}\right)\right) \cdot \Pi\left(A_{\alpha(j)}\right),
$$

with $f\left(x_{\alpha(0)}\right)=0$ by convention, and

$$
\int^{\mathcal{S}} f \mathrm{~d} \Pi=\bigvee_{j=1}^{n}\left(f\left(x_{\alpha(j)}\right) \wedge \Pi\left(A_{\alpha(j)}\right)\right) .
$$


As shown in [17], the Sugeno integral for possibility measures can be alternatively defined as follows:

$$
\int^{\mathcal{S}} f \mathrm{~d} \Pi=\bigvee_{x \in X}(f(x) \wedge \pi(x)),
$$

where $\pi$ is the possibility distribution associated to $\Pi$.

Possibility measures were originally introduced by Zadeh in [50]. Since then, they have been studied as measures of uncertainty capable of representing incomplete information and as models of partial belief offering both a qualitative and a numerical alternative to probabilities [20]. Similar to probabilities, possibility measures lend themselves to several different interpretations. The epistemic interpretation is one of the most prominent ones and sees possibilities as a measure of plausibility of the occurrence of an event. In this context, a possibility distribution over a set of alternatives can be seen as a way to rank how plausible or likely each option is. Possibility can also be seen as a measure of logical consistency. The possibility of a proposition is a measure of how consistent it is with the available information. Another interpretation of possibilities is that of measures of feasibility: they rank how different options are easy to achieve. Finally, possibility measures can be cast in a deontic framework and be seen as a way to measure permissibility to evaluate the degree to which an action is allowed or permitted.

In this work our view of possibility remains neutral and we do not take any stance in regards to its interpretation. As mentioned above, we simply see a possibility distribution as a way to formalise a different notion of randomisation.

\section{Possibilistic Mixed Extensions}

In this section, we introduce the notion of a possibilistic mixed extension, i.e. extensions of strategic-form games where players are equipped with the set of all possibility distributions over their strategy space. Each player will have a certain expectation on their outcome based on their and the other players' distributions. We offer two distinct approaches to model players' expectation: a quantitative approach, based on the Choquet integral, and a qualitative one, based on the Sugeno integral. Again, it is worth pointing out that we are not making any assumptions concerning the interpretation of possibility distributions here (later, in Section 4, they will be seen as a measure of commitment for a player to make certain choices). The results we present in this section are independent of any interpretation.

Definition 9 (Possibilistic Mixed Strategy). Given a strategic-form game G, a possibilistic mixed strategy for a player $i \in\{1,2\}$ is a possibility distribution $\pi_{i}: S_{i} \rightarrow[0,1]$.

We denote by $\Sigma_{i}$ the set of all possibilistic mixed strategies of player $i$.

The possibilistic mixed strategies of the players will have an effect on the game and on the players' expectations concerning the outcome. A player's expectation will depend not only on their own strategy but also on the other player's. For this, we need a notion of joint distribution. Given a pair of possibility distributions $\left(\pi_{1}, \pi_{2}\right)$ (one for each player) we define the joint possibilistic mixed strategy of $\left(\pi_{1}, \pi_{2}\right)$ as the joint possibility distribution

$$
\pi_{\times}: S_{1} \times S_{2} \rightarrow[0,1]
$$

such that for each pair $\left(s_{1}, s_{2}\right) \in S_{1} \times S_{2}$

$$
\pi_{\times}\left(s_{1}, s_{2}\right)=\pi_{1}\left(s_{1}\right) \wedge \pi_{2}\left(s_{2}\right) .
$$


It is worth pointing out that for the definition of a joint distribution we could have chosen any triangular norm (see, e.g. [36]) to combine $\pi_{1}$ and $\pi_{2} \cdot{ }^{1}$ The choice of the minimum function $\wedge$ is a standard choice in the framework of possibility theory and also works both with the quantitative and qualitative approaches of Choquet and Sugeno integration.

In order to study equilibrium concepts based on possibility distributions, it seems natural to adopt a notion of expectation for possibility measures. The two most common approaches towards defining expectation for possibility measures are based on the Choquet and the Sugeno integral [28]. The next two subsections introduce and analyse a concept of possibilistic mixed extensions of games where players' expectation is based on these two different notions.

\subsection{Quantitative Approach: Choquet Expectation}

We start by defining the notion of Choquet expectation.

Take a strategic-form game $\mathbf{G}$ with set of strategy combinations,

$$
S_{1} \times S_{2}=\left\{\left(s_{1}, s_{2}\right)_{1}, \ldots,\left(s_{1}, s_{2}\right)_{m}\right\}
$$

and take a permutation $\alpha_{i}$, for each $i \in\{1,2\}$, on $S_{1} \times S_{2}$ such that

$$
u_{i}\left(\left(s_{1}, s_{2}\right)_{\alpha_{i}(1)}\right) \leq u_{i}\left(\left(s_{1}, s_{2}\right)_{\alpha_{i}(2)}\right) \leq \cdots \leq u_{i}\left(\left(s_{1}, s_{2}\right)_{\alpha_{i}(m)}\right) .
$$

Let $A_{\alpha_{i}(j)} \subseteq S_{1} \times S_{2}$ be defined as

$$
A_{\alpha_{i}(j)}=\left\{\left(s_{1}, s_{2}\right)_{\alpha_{i}(j)},\left(s_{1}, s_{2}\right)_{\alpha_{i}(j+1)}, \ldots,\left(s_{1}, s_{2}\right)_{\alpha_{i}(m)}\right\},
$$

with $i \in\{1,2\}$ and $1 \leq j \leq m$.

Let $\left(\pi_{1}, \pi_{2}\right) \in \Sigma_{1} \times \Sigma_{2}$ be a pair of possibility distributions for $\mathbf{G}$. Given the joint distribution $\pi_{\times}$on $S_{1} \times S_{2}$, let $\Pi$ be the possibility measure generated from $\pi_{\times}$. The Choquet expectation of player $i$ is the Choquet integral of the utility function $u_{i}$ with respect to the possibility measure $\Pi$ and is defined as

$$
E_{i}^{\mathcal{C} h}\left(\pi_{1}, \pi_{2}\right)=\int^{\mathcal{C} h} u_{i} \mathrm{~d} \Pi=\sum_{j=1}^{m}\left(u_{i}\left(\left(s_{1}, s_{2}\right)_{\alpha_{i}(j)}\right)-u_{i}\left(\left(s_{1}, s_{2}\right)_{\alpha_{i}(j-1)}\right)\right) \cdot \Pi\left(A_{\alpha_{i}(j)}\right),
$$

where

$$
\Pi\left(A_{\alpha_{i}(j)}\right)=\bigvee_{\left(s_{1}, s_{2}\right) \in A_{\alpha_{i}(j)}}\left(\pi_{1}\left(s_{1}\right) \wedge \pi_{2}\left(s_{2}\right)\right),
$$

and $u_{i}\left(\left(s_{1}, s_{2}\right)_{\alpha_{i}(0)}\right)=0$, by convention.

We now introduce the possibilistic counterpart of Definition 5 as well as specific notions of best response and equilibrium for Choquet integrals w.r.t. possibility measures.

\footnotetext{
${ }^{1} \mathrm{~A}$ triangular norm [36] is a binary function $*:[0,1]^{2} \rightarrow[0,1]$ that is commutative, associative, monotone and has 1 as a neutral element.
} 
Definition 10 (Choquet Mixed Extension). Let

$$
\mathbf{G}=\left\langle N, S_{1}, S_{2}, u_{1}, u_{2}\right\rangle
$$

be a strategic-form game. The Choquet mixed extension of $\mathbf{G}$ is the game

$$
\mathfrak{G}^{C h}=\left\langle N, \Sigma_{1}, \Sigma_{2}, e u_{1}, e u_{2}\right\rangle
$$

where, for $i \in\{1,2\}$ :

- Each $\Sigma_{i}$ is the set of all possibilistic mixed strategies of player $i$ over $S_{i}$.

- Each eu $: \Sigma_{1} \times \Sigma_{2} \rightarrow \mathbb{R}$ is a payoff function that associates with each possibilistic mixed strategy combination $\left(\pi_{1}, \pi_{2}\right)$ its Choquet expected utility

$$
e u_{i}\left(\pi_{1}, \pi_{2}\right)=E_{i}^{\mathcal{C h}}\left(\pi_{1}, \pi_{2}\right) .
$$

Definition 11 (Best Response: Choquet). Let $\mathbf{G}$ be a strategic-form game and $\mathfrak{G}^{\text {Ch }}$ be its Choquet mixed extension. Player 1's best response to a possibilistic mixed strategy $\pi_{2} \in \Sigma_{2}$ is a possibility distribution $\pi_{1} \in \Sigma_{1}$ such that, for all $\pi_{1}^{\prime} \in \Sigma_{1}$ :

$$
E_{1}^{\mathcal{C h}}\left(\pi_{1}, \pi_{2}\right) \geq E_{1}^{\mathcal{C h}}\left(\pi_{1}^{\prime}, \pi_{2}\right) .
$$

The definition for Player 2 is analogous.

Definition 12 (Choquet Equilibrium). Let $\mathbf{G}$ be a strategic-form game and $\mathfrak{G}^{\text {Ch }}$ be its Choquet mixed extension. We call a pair of possibilistic mixed strategies $\left(\pi_{1}, \pi_{2}\right) \in \Sigma_{1} \times \Sigma_{2}$ a Choquet equilibrium for $\mathbf{G}$ (CE, for short) if each player's possibilistic mixed strategy is a best response to the other player's possibilistic mixed strategy.

For a strategic-form game $\mathbf{G}$, we denote by $\mathcal{C} h(\mathbf{G})$ its set of Choquet equilibria.

We now explore the existence of CEs for games with possibilistic mixed strategies.

Theorem 13. Let $\mathbf{G}$ and $\mathfrak{G}^{C h}$ be a strategic-form game and its Choquet mixed extension, respectively. Let $\left(\pi_{1}, \pi_{2}\right) \in \Sigma_{1} \times \Sigma_{2}$. For each player $i=\{1,2\}$ and $1 \leq j \leq m$ let

$$
A_{\alpha_{i}(j)}^{\max }=\left\{\left(s_{1}, s_{2}\right) \mid\left(s_{1}, s_{2}\right) \in A_{\alpha_{i}(j)} \text { and } s_{-i} \in \underset{s_{-i}^{\prime} \in S_{-i},\left(s_{1}^{\prime}, s_{2}^{\prime}\right) \in A_{\alpha_{i}(j)}}{\operatorname{argmax}} \pi_{-i}\left(s_{-i}^{\prime}\right)\right\} .
$$

Then, the following conditions are equivalent:

1. $\left(\pi_{1}, \pi_{2}\right)$ is a Choquet equilibrium for $\mathbf{G}$.

2. For every $1 \leq j \leq m$ such that $u_{1}\left(\left(s_{1}, s_{2}\right)_{\alpha_{1}(j)}\right)-u_{1}\left(\left(s_{1}, s_{2}\right)_{\alpha_{1}(j-1)}\right)>0$, there exists $\left(s_{1}, s_{2}\right) \in A_{\alpha_{1}(j)}^{\max }$ such that

$$
\pi_{1}\left(s_{1}\right) \geq \pi_{2}\left(s_{2}\right)
$$

and for every $1 \leq j \leq m$ such that $u_{2}\left(\left(s_{1}, s_{2}\right)_{\alpha_{2}(j)}\right)-u_{2}\left(\left(s_{1}, s_{2}\right)_{\alpha_{2}(j-1)}\right)>0$ there exists $\left(s_{1}^{\prime}, s_{2}^{\prime}\right) \in A_{\alpha_{2}(j)}^{\max }$ such that

$$
\pi_{2}\left(s_{2}^{\prime}\right) \geq \pi_{1}\left(s_{1}^{\prime}\right)
$$


Proof. (1) $\Rightarrow(2)$ : Suppose (2) does not hold. Without any loss of generality we can assume that for player 1 and for some $j$ such that $u_{1}\left(\left(s_{1}, s_{2}\right)_{\alpha_{1}(j)}\right)-u_{1}\left(\left(s_{1}, s_{2}\right)_{\alpha_{1}(j-1)}\right)>0$, for all $\left(s_{1}, s_{2}\right) \in A_{\alpha_{1}(j)}^{\max }$

$$
\pi_{1}\left(s_{1}\right)<\pi_{2}\left(s_{2}\right) .
$$

Choose a strategy combination $\left(s_{1}^{\prime}, s_{2}^{\prime}\right) \in A_{\alpha_{1}(j)}^{\max }$, and take a new possibility distribution $\pi_{1}^{\prime}$ for player 1 such that, for all $s_{1} \in S_{1} \backslash\left\{s_{1}^{\prime}\right\}$,

$$
\pi_{1}^{\prime}\left(s_{1}\right)=\pi_{1}\left(s_{1}\right) \quad \text { and } \quad \pi_{1}^{\prime}\left(s_{1}^{\prime}\right)=\pi_{2}\left(s_{2}^{\prime}\right),
$$

i.e.: $\pi_{1}^{\prime}$ differs from $\pi_{1}$ only in the value assigned to the strategy $s_{1}^{\prime}$, so that

$$
\pi_{1}^{\prime}\left(s_{1}^{\prime}\right)>\pi_{1}\left(s_{1}^{\prime}\right) .
$$

Let $\Pi^{\prime}$ be the possibility measure generated by the joint possibility distribution $\pi_{\times}^{\prime}$ obtained from $\pi_{1}^{\prime}$ and $\pi_{2}$. Then, for each $1 \leq j^{\prime} \leq m$, with $j^{\prime} \neq j$,

$$
\Pi\left(A_{\alpha_{1}\left(j^{\prime}\right)}\right)=\underset{\left(s_{1}, s_{2}\right) \in A_{\alpha_{1}\left(j^{\prime}\right)}}{\bigvee}\left(\pi_{1}\left(s_{1}\right) \wedge \pi_{2}\left(s_{2}\right)\right) \leq \underset{\left(s_{1}, s_{2}\right) \in A_{\alpha_{1}\left(j^{\prime}\right)}}{\bigvee}\left(\pi_{1}^{\prime}\left(s_{1}\right) \wedge \pi_{2}\left(s_{2}\right)\right)=\Pi^{\prime}\left(A_{\alpha_{1}\left(j^{\prime}\right)}\right)
$$

and, for $j$,

$$
\begin{aligned}
& \Pi\left(A_{\alpha_{1}(j)}\right)=\bigvee_{\left(s_{1}, s_{2}\right) \in A_{\alpha_{1}(j)}}\left(\pi_{1}\left(s_{1}\right) \wedge \pi_{2}\left(s_{2}\right)\right)<\pi_{2}\left(s_{2}\right), \\
& \Pi^{\prime}\left(A_{\alpha_{1}(j)}\right)=\bigvee_{\left(s_{1}, s_{2}\right) \in A_{\alpha_{1}(j)}}\left(\pi_{1}^{\prime}\left(s_{1}\right) \wedge \pi_{2}\left(s_{2}\right)\right)=\pi_{2}\left(s_{2}\right) .
\end{aligned}
$$

Consequently

$$
E_{1}^{\mathcal{C h}}\left(\pi_{1}, \pi_{2}\right)<E_{1}^{\mathcal{C h}}\left(\pi_{1}^{\prime}, \pi_{2}\right),
$$

and so $\left(\pi_{1}, \pi_{2}\right)$ is not an equilibrium and (1) does not hold.

$(2) \Rightarrow(1)$ : Suppose that (2) holds. Then, for player 1 , and all $\pi_{1}^{\prime} \in \Sigma_{1}$, for each $j$ such that $u_{1}\left(\left(s_{1}, s_{2}\right)_{\alpha_{1}(j)}\right)-u_{1}\left(\left(s_{1}, s_{2}\right)_{\alpha_{1}(j-1)}\right)>0$,

$$
\begin{aligned}
\Pi^{\prime}\left(A_{\alpha_{1}(j)}\right)= & \bigvee_{\left(s_{1}^{\prime \prime}, s_{2}^{\prime \prime}\right) \in A_{\alpha_{1}(j)}}\left(\pi_{1}^{\prime}\left(s_{1}^{\prime \prime}\right) \wedge \pi_{2}\left(s_{2}^{\prime \prime}\right)\right) \leq \pi_{2}\left(s_{2}\right)= \\
& \bigvee_{\left(s_{1}^{\prime \prime}, s_{2}^{\prime \prime}\right) \in A_{\alpha_{1}(j)}}\left(\pi_{1}\left(s_{1}^{\prime \prime}\right) \wedge \pi_{2}\left(s_{2}^{\prime \prime}\right)\right)=\Pi\left(A_{\alpha_{1}(j)}\right) .
\end{aligned}
$$

So, we have that

$$
E_{1}^{\mathcal{C h}}\left(\pi_{1}^{\prime}, \pi_{2}\right) \leq E_{1}^{\mathcal{C h}}\left(\pi_{1}, \pi_{2}\right),
$$

A similar argument holds for player 2 , and so $\left(\pi_{1}, \pi_{2}\right)$ is a CE.

The following corollary is an immediate consequence of the previous result.

Corollary 14. Every strategic-form game $\mathbf{G}$ admits a Choquet equilibrium, i.e. $\mathcal{C} h(\mathbf{G}) \neq \emptyset$. In particular, every $\left(\pi_{1}, \pi_{2}\right) \in \Sigma_{1} \times \Sigma_{2}$ is an equilibrium whenever, for every $i$ and every $s_{i} \in S_{i}$, $\pi_{i}\left(s_{i}\right)=1$. 
Proof. It is easy to see that the above case satisfies condition (2) of Theorem 13.

The set of CEs for strategic-form games is invariant under affine transformations.

Definition 15. Two strategic-form games

$$
\mathbf{G}_{1}=\left\{N, S_{1}, S_{2}, u_{1}, u_{2}\right\} \quad \mathbf{G}_{2}=\left\{N, S_{1}, S_{2}, v_{1}, v_{2}\right\}
$$

with the same sets of pure strategies are strategically equivalent if, for each player $i$, the function $u_{i}$ is a positive affine transformation of the function $v_{i}$ : i.e., there exist $a_{i}>0$ and $b_{i} \in \mathbb{R}$ such that

$$
v_{i}\left(s_{1}, s_{2}\right)=a_{i} \cdot u_{i}\left(s_{1}, s_{2}\right)+b_{i}
$$

for all $\left(s_{1}, s_{2}\right) \in S_{1} \times S_{2}$.

Proposition 16. Let $\mathbf{G}_{1}$ and $\mathbf{G}_{2}$ be two strategically equivalent strategic-form games. Then

$$
\mathcal{C} h\left(\mathbf{G}_{1}\right)=\mathcal{C} h\left(\mathbf{G}_{2}\right),
$$

i.e. both games have the same set of Choquet equilibria.

Proof. The proof is easy. Suppose that a pair $\left(\pi_{1}, \pi_{2}\right)$ is a $\mathrm{CE}$ for $\mathbf{G}_{1}$ but not for $\mathbf{G}_{2}$. Then, without any loss of generality, we can assume there exists a distribution $\pi_{1}^{\prime} \in \Sigma_{1}$ such that

$$
\begin{aligned}
& \sum_{j=1}^{m}\left(v_{1}\left(\left(s_{1}, s_{2}\right)_{\alpha_{1}(j)}\right)-v_{1}\left(\left(s_{1}, s_{2}\right)_{\alpha_{1}(j-1)}\right)\right) \cdot \Pi\left(A_{\alpha_{1}(j)}\right)< \\
& \sum_{j=1}^{m}\left(v_{1}\left(\left(s_{1}, s_{2}\right)_{\alpha_{1}(j)}\right)-v_{1}\left(\left(s_{1}, s_{2}\right)_{\alpha_{1}(j-1)}\right)\right) \cdot \Pi^{\prime}\left(A_{\alpha_{1}(j)}\right),
\end{aligned}
$$

where $\Pi$ and $\Pi^{\prime}$ are the possibility measures derived from the joint distribution of $\pi_{1}$ and $\pi_{2}$, and $\pi_{1}^{\prime}$ and $\pi_{2}$, respectively.

From the fact that

$$
v_{1}\left(s_{1}, s_{2}\right)=a_{1} \cdot u_{1}\left(s_{1}, s_{2}\right)+b_{1}
$$

we easily obtain

$$
\begin{aligned}
& \sum_{j=1}^{m}\left(u_{1}\left(\left(s_{1}, s_{2}\right)_{\alpha_{1}(j)}\right)-u_{1}\left(\left(s_{1}, s_{2}\right)_{\alpha_{1}(j-1)}\right)\right) \cdot \Pi\left(A_{\alpha_{1}(j)}\right)< \\
& \sum_{j=1}^{m}\left(u_{1}\left(\left(s_{1}, s_{2}\right)_{\alpha_{1}(j)}\right)-u_{1}\left(\left(s_{1}, s_{2}\right)_{\alpha_{1}(j-1)}\right)\right) \cdot \Pi^{\prime}\left(A_{\alpha_{1}(j)}\right),
\end{aligned}
$$

which means that $\left(\pi_{1}, \pi_{2}\right)$ is not a $\mathrm{CE}$ for $\mathbf{G}_{1}$, contradicting the original assumption.

The converse can be proved with a similar argument.

Theorem 13 implicitly gives us a polynomial-time algorithm to check whether two possibility distributions form a CE.

Proposition 17. Let $\mathbf{G}$ and $\mathfrak{G}^{C h}$ be a strategic-form game and its Choquet mixed extension, respectively. Checking whether any $\left(\pi_{1}, \pi_{2}\right) \in \Sigma_{1} \times \Sigma_{2}$ is a Choquet equilibrium for $\mathbf{G}$ is in PTIME. 
Proof. The input is an explicit representation of the game $\mathbf{G}$ containing, for each player, the value of their utility function at each strategy combination, along with the distributions $\pi_{1}$ and $\pi_{2}$. The algorithm proceeds as follows.

For player 1, order the elements of $S_{1} \times S_{2}$ in terms of their utility, obtaining

$$
u_{1}\left(\left(s_{1}, s_{2}\right)_{\alpha_{1}(1)}\right) \leq u_{1}\left(\left(s_{1}, s_{2}\right)_{\alpha_{1}(2)}\right) \leq \cdots \leq u_{1}\left(\left(s_{1}, s_{2}\right)_{\alpha_{1}(m)}\right) .
$$

Then, for each $1 \leq j \leq m$, if $u_{1}\left(\left(s_{1}, s_{2}\right)_{\alpha_{1}(j)}\right)-u_{1}\left(\left(s_{1}, s_{2}\right)_{\alpha_{1}(j-1)}\right)>0$ define the sets

$$
A_{\alpha_{1}(j)} \quad \text { and } \quad A_{\alpha_{1}(j)}^{\max },
$$

and check

$$
\text { if there is any }\left(s_{1}, s_{2}\right) \in A_{\alpha_{1}(j)}^{\max } \text { such that } \pi_{1}\left(s_{1}\right) \geq \pi_{2}\left(s_{2}\right) \text {. }
$$

If for any $j$ the answer to $(\sharp)$ is negative, the algorithm stops and $\left(\pi_{1}, \pi_{2}\right)$ is not an equilibrium. If not, the process is repeated for player 2, i.e.: order the set of strategy combination in terms on player 2's utility, and for each $1 \leq j \leq m$, if $u_{2}\left(\left(s_{1}, s_{2}\right)_{\alpha_{2}(j)}\right)-u_{2}\left(\left(s_{1}, s_{2}\right)_{\alpha_{2}(j-1)}\right)>0$, define the sets

$$
A_{\alpha_{2}(j)} \quad \text { and } \quad A_{\alpha_{2}(j)}^{\max },
$$

and check

$$
(\sharp \sharp) \quad \text { if there is any }\left(s_{1}, s_{2}\right) \in A_{\alpha_{2}(j)}^{\max } \text { such that } \pi_{2}\left(s_{2}\right) \geq \pi_{1}\left(s_{1}\right) \text {. }
$$

If for any $j$ the answer to $(\sharp \sharp)$ is negative, the algorithm stops and $\left(\pi_{1}, \pi_{2}\right)$ is not an equilibrium. If not, the process reaches the end and $\left(\pi_{1}, \pi_{2}\right)$ is a $\mathrm{CE}$.

It is clear that both the definition of $A_{\alpha_{i}(j)}$ and $A_{\alpha_{i}(j)}^{\max }$ for each player $i$ and checking the conditions $(\sharp)$ and $(\sharp \sharp)$ can be computed in time polynomial in the size of the input.

\subsection{Qualitative Approach: Sugeno Expectation}

We start by defining the notion of Sugeno expectation for strategic-form games. Following the definition of Sugeno integration given in Section 2, when dealing with the expected utility with respect to a possibility measure, we assume the range of a utility function $u$ to be a subset of $[0,1]$ containing both 0 and 1 (see [6]).

Remark 18. From now on, whenever we deal with Sugeno expected utility, we assume strategicform games to have utility functions $u_{i}, i \in\{1,2\}$, such that

$$
\operatorname{ran}\left(u_{i}\right)=\left\{0, a_{1}, \ldots, a_{m_{i}-2}, 1\right\} \subset[0,1] .
$$

Take a strategic-form game $\mathbf{G}$ with set of strategy combinations,

$$
S_{1} \times S_{2}=\left\{\left(s_{1}, s_{2}\right)_{1}, \ldots,\left(s_{1}, s_{2}\right)_{m}\right\}
$$

and take a permutation $\alpha_{i}$, for each $i \in\{1,2\}$, on $S_{1} \times S_{2}$ such that

$$
u_{i}\left(\left(s_{1}, s_{2}\right)_{\alpha_{i}(1)}\right) \leq u_{i}\left(\left(s_{1}, s_{2}\right)_{\alpha_{i}(2)}\right) \leq \cdots \leq u_{i}\left(\left(s_{1}, s_{2}\right)_{\alpha_{i}(m)}\right) .
$$


Let $A_{\alpha_{i}(j)} \subseteq S_{1} \times S_{2}$ be defined as

$$
A_{\alpha_{i}(j)}=\left\{\left(s_{1}, s_{2}\right)_{\alpha_{i}(j)},\left(s_{1}, s_{2}\right)_{\alpha_{i}(j+1)}, \ldots,\left(s_{1}, s_{2}\right)_{\alpha_{i}(m)}\right\},
$$

with $i \in\{1,2\}$ and $1 \leq j \leq m$.

Let $\left(\pi_{1}, \pi_{2}\right) \in \Sigma_{1} \times \Sigma_{2}$ be a pair of possibilistic mixed strategies for $\mathbf{G}$. Given their joint distribution $\pi_{\times}$on $S_{1} \times S_{2}$, let $\Pi$ be the possibility measure generated from $\pi_{\times}$. The Sugeno expectation of player $i$ is the Sugeno integral of the utility function $u_{i}$ with respect to the possibility measure $\Pi$ and is defined as

$$
E_{i}^{\mathcal{S}}\left(\pi_{1}, \pi_{2}\right)=\int^{\mathcal{S}} u_{i} \mathrm{~d} \Pi=\bigvee_{j=1}^{m}\left(u_{i}\left(\left(s_{1}, s_{2}\right)_{\alpha_{i}(j)}\right) \wedge \Pi\left(A_{\alpha_{i}(j)}\right)\right) .
$$

Given [17], $E_{i}^{\mathcal{S}}\left(\pi_{1}, \pi_{2}\right)$ can be equivalently written in the following form:

$$
E_{i}^{\mathcal{S}}\left(\pi_{1}, \pi_{2}\right)=\int^{\mathcal{S}} u_{i} \mathrm{~d} \Pi=\bigvee_{\left(s_{1}, s_{2}\right) \in S_{1} \times S_{2}}\left(u_{i}\left(\left(s_{1}, s_{2}\right)\right) \wedge\left(\pi_{1}\left(s_{1}\right) \wedge \pi_{2}\left(s_{2}\right)\right)\right) .
$$

We now introduce the possibilistic counterpart of Definition 5 as well as specific notions of best response and equilibrium for Sugeno integrals w.r.t. possibility distributions.

Definition 19 (Sugeno Mixed Extension). Let

$$
\mathbf{G}=\left\langle N, S_{1}, S_{2}, u_{1}, u_{2}\right\rangle
$$

be a strategic-form game. The Sugeno mixed extension of $\mathbf{G}$ is the game

$$
\mathfrak{G}^{S}=\left\langle N, \Sigma_{1}, \Sigma_{2}, e u_{1}, e u_{2}\right\rangle
$$

where, for $i \in\{1,2\}$ :

- Each $\Sigma_{i}$ is the set of all possibilistic mixed strategies of player $i$ over $S_{i}$.

- Each eu $: \Sigma_{1} \times \Sigma_{2} \rightarrow \mathbb{R}$ is a payoff function that associates with each possibilistic mixed strategy combination $\left(\pi_{1}, \pi_{2}\right)$ its Sugeno expected utility

$$
e u_{i}\left(\pi_{1}, \pi_{2}\right)=E_{i}^{\mathcal{S}}\left(\pi_{1}, \pi_{2}\right) .
$$

Definition 20 (Best Response: Sugeno). Let $\mathbf{G}$ be a strategic-form game and $\mathfrak{G}^{S}$ be its Sugeno mixed extension. Player 1's best response to a possibilistic mixed strategy $\pi_{2} \in \Sigma_{2}$ is a possibility distribution $\pi_{1} \in \Sigma_{1}$ such that, for all $\pi_{1}^{\prime} \in \Sigma_{1}$ :

$$
E_{1}^{\mathcal{S}}\left(\pi_{1}, \pi_{2}\right) \geq E_{1}^{\mathcal{S}}\left(\pi_{1}^{\prime}, \pi_{2}\right) .
$$

The definition for Player 2 is analogous.

Definition 21 (Sugeno Equilibrium). Let $\mathbf{G}$ be a strategic-form game and $\mathfrak{G}^{S}$ be its Sugeno mixed extension. We call a pair of possibilistic mixed strategies $\left(\pi_{1}, \pi_{2}\right) \in \Sigma_{1} \times \Sigma_{2}$ a Sugeno equilibrium for $\mathbf{G}$ (SE, for short) if each player's possibilistic mixed strategy is a best response to the other player's possibilistic mixed strategy. 
For a strategic-form game $\mathbf{G}$, we denote by $\mathcal{S}(\mathbf{G})$ the set of its Sugeno equilibria.

We now give a characterisation of the conditions for a pair of possibilistic mixed strategies to be a Sugeno equilibrium.

Theorem 22. Let $\mathbf{G}$ and $\mathfrak{G}^{S}$ be a strategic-form game and its Sugeno mixed extension, respectively. Let $\left(\pi_{1}, \pi_{2}\right) \in \Sigma_{1} \times \Sigma_{2}$ and let

$$
B_{1}=\underset{\left(s_{1}, s_{2}\right) \in S_{1} \times S_{2}}{\operatorname{argmax}}\left(\pi_{2}\left(s_{2}\right) \wedge u_{1}\left(s_{1}, s_{2}\right)\right) \quad \text { and } \quad B_{2}=\underset{\left(s_{1}, s_{2}\right) \in S_{1} \times S_{2}}{\operatorname{argmax}}\left(\pi_{1}\left(s_{1}\right) \wedge u_{2}\left(s_{1}, s_{2}\right)\right) .
$$

Then the following conditions are equivalent:

1. $\left(\pi_{1}, \pi_{2}\right)$ is a Sugeno equilibrium for $\mathbf{G}$.

2. There exists $\left(s_{1}, s_{2}\right) \in B_{1}$ such that

$$
\pi_{1}\left(s_{1}\right) \geq \pi_{2}\left(s_{2}\right) \wedge u_{1}\left(s_{1}, s_{2}\right)
$$

and there exists $\left(s_{1}, s_{2}\right) \in B_{2}$ such that

$$
\pi_{2}\left(s_{2}\right) \geq \pi_{1}\left(s_{1}\right) \wedge u_{2}\left(s_{1}, s_{2}\right) .
$$

Proof. (1) $\Rightarrow(2)$ : Suppose (2) does not hold. Without any loss of generality, we can assume that for player 1 and all $\left(s_{1}, s_{2}\right) \in B_{1}$,

$$
\pi_{1}\left(s_{1}\right)<\pi_{2}\left(s_{2}\right) \wedge u_{1}\left(s_{1}, s_{2}\right)
$$

Moreover, it is clear that, for all $\left(s_{1}, s_{2}\right) \in B_{1}$,

$$
E_{1}^{\mathcal{S}}\left(\pi_{1}, \pi_{2}\right)<\pi_{2}\left(s_{2}\right) \wedge u_{1}\left(s_{1}, s_{2}\right) .
$$

Choose a strategy combination $\left(s_{1}^{\prime}, s_{2}^{\prime}\right) \in B_{1}$ and take a new possibility distribution $\pi_{1}^{\prime} \in \Sigma_{1}$ for player 1 such that, for all $s_{1} \in S_{1} \backslash\left\{s_{1}^{\prime}\right\}$,

$$
\pi_{1}^{\prime}\left(s_{1}\right)=\pi_{1}\left(s_{1}\right) \quad \text { and } \quad \pi_{1}^{\prime}\left(s_{1}^{\prime}\right)=\pi_{2}\left(s_{2}^{\prime}\right) \wedge u_{1}\left(s_{1}^{\prime}, s_{2}^{\prime}\right),
$$

i.e.: $\pi_{1}^{\prime}$ differs from $\pi_{1}$ only in the value assigned to the strategy $s_{1}^{\prime}$, so that

$$
\pi_{1}^{\prime}\left(s_{1}^{\prime}\right)>\pi_{1}\left(s_{1}^{\prime}\right)
$$

It is clear that, by construction,

$$
E_{1}^{\mathcal{S}}\left(\pi_{1}^{\prime}, \pi_{2}\right)=\pi_{2}\left(s_{2}^{\prime}\right) \wedge u_{1}\left(s_{1}^{\prime}, s_{2}^{\prime}\right),
$$

and so,

$$
E_{1}^{\mathcal{S}}\left(\pi_{1}, \pi_{2}\right)<\pi_{2}\left(s_{2}^{\prime}\right) \wedge u_{1}\left(s_{1}^{\prime}, s_{2}^{\prime}\right)=E_{1}^{\mathcal{S}}\left(\pi_{1}^{\prime}, \pi_{2}\right) .
$$

Then $\left(\pi_{1}, \pi_{2}\right)$ is not a Sugeno equilibrium and (1) does not hold.

$(2) \Rightarrow(1)$ : Suppose that $(2)$ holds and let $\left(s_{1}, s_{2}\right) \in B_{1}$ be such that

$$
\pi_{1}\left(s_{1}\right) \geq \pi_{2}\left(s_{2}\right) \wedge u_{1}\left(s_{1}, s_{2}\right) \text {. }
$$


Given that $\left(s_{1}, s_{2}\right) \in B_{1}$, then, for all $\left(s_{1}^{\prime}, s_{2}^{\prime}\right)$,

$$
\pi_{2}\left(s_{2}^{\prime}\right) \wedge u_{1}\left(s_{1}^{\prime}, s_{2}^{\prime}\right) \leq \pi_{2}\left(s_{2}\right) \wedge u_{1}\left(s_{1}, s_{2}\right),
$$

and so

$$
\pi_{1}\left(s_{1}^{\prime}\right) \wedge \pi_{2}\left(s_{2}^{\prime}\right) \wedge u_{1}\left(s_{1}^{\prime}, s_{2}^{\prime}\right) \leq \pi_{2}\left(s_{2}\right) \wedge u_{1}\left(s_{1}, s_{2}\right)=\pi_{1}\left(s_{1}\right) \wedge \pi_{2}\left(s_{2}\right) \wedge u_{1}\left(s_{1}, s_{2}\right),
$$

and

$$
E_{1}^{\mathcal{S}}\left(\pi_{1}, \pi_{2}\right)=\pi_{1}\left(s_{1}\right) \wedge \pi_{2}\left(s_{2}\right) \wedge u_{1}\left(s_{1}, s_{2}\right) .
$$

Also, for any distribution $\pi_{1}^{\prime}$ and any $\left(s_{1}^{\prime}, s_{2}^{\prime}\right)$ we have that

$$
\pi_{1}^{\prime}\left(s_{1}^{\prime}\right) \wedge \pi_{2}\left(s_{2}^{\prime}\right) \wedge u_{1}\left(s_{1}^{\prime}, s_{2}^{\prime}\right) \leq \pi_{2}\left(s_{2}\right) \wedge u_{1}\left(s_{1}, s_{2}\right)
$$

and so, for all $\pi_{1}^{\prime}$

$$
E_{1}^{\mathcal{S}}\left(\pi_{1}^{\prime}, \pi_{2}\right) \leq E_{1}^{\mathcal{S}}\left(\pi_{1}, \pi_{2}\right) .
$$

A similar argument can be made for any $\left(s_{1}, s_{2}\right) \in B_{2}$ such that

$$
\pi_{2}\left(s_{2}\right) \geq \pi_{1}\left(s_{1}\right) \wedge u_{2}\left(s_{1}, s_{2}\right)
$$

Therefore, $\left(\pi_{1}, \pi_{2}\right)$ is a Sugeno a equilibrium.

The following corollary similar to Corollary 14 is an immediate consequence of the previous result.

Corollary 23. Every strategic-form game $\mathbf{G}$ admits a Sugeno equilibrium, i.e. $\mathcal{S}(\mathbf{G}) \neq \emptyset$. In particular, every $\left(\pi_{1}, \pi_{2}\right) \in \Sigma_{1} \times \Sigma_{2}$ is an equilibrium whenever, for every $i$ and every $s_{i} \in S_{i}$, $\pi_{i}\left(s_{i}\right)=1$.

In the previous subsection, we defined two games to be strategically equivalent whenever the utility functions of one of them are an affine transformation of the functions of the other. Proposition 16 shows that strategically equivalent strategic-form games share the same Choquet equilibria. This clearly does not apply to Sugeno equilibria, the obvious reason being the fact that we require the utility functions $u_{i}, i \in\{1,2\}$, to be such that

$$
\operatorname{ran}\left(u_{i}\right)=\left\{0, a_{1}, \ldots, a_{m_{i}-2}, 1\right\} \subset[0,1] .
$$

Theorem 22 implicitly gives us a polynomial-time algorithm to check whether two possibilistic mixed strategies form a SE.

Proposition 24. Let $\mathbf{G}$ and $\mathfrak{G}^{S}$ be a strategic-form game and its Sugeno mixed extension, respectively. Checking whether any $\left(\pi_{1}, \pi_{2}\right) \in \Sigma_{1} \times \Sigma_{2}$ is a Sugeno equilibrium for $\mathbf{G}$ is in PTIME.

Proof. The input is an explicit representation of the game $\mathbf{G}$ containing, for each player, the value of their utility function at each strategy combination, along with the distributions $\pi_{1}$ and $\pi_{2}$. The algorithm proceeds as follows.

For player 1 define the set $B_{1}$, and check if there is any

$$
\left(s_{1}, s_{2}\right) \in B_{1}
$$


such that

$$
\pi_{1}\left(s_{1}\right) \geq \pi_{2}\left(s_{2}\right) \wedge u_{1}\left(s_{1}, s_{2}\right) .
$$

For player 2 define the set $B_{2}$, and check if there is any

$$
\left(s_{1}, s_{2}\right) \in B_{2}
$$

such that

$$
\pi_{2}\left(s_{2}\right) \geq \pi_{1}\left(s_{2}\right) \wedge u_{2}\left(s_{1}, s_{2}\right) .
$$

If the answer to either (1) or $(2)$ is negative, the algorithm stops and $\left(\pi_{1}, \pi_{2}\right)$ is not an equilibrium. Otherwise, $\left(\pi_{1}, \pi_{2}\right)$ is a SE.

It is clear that both sets $B_{1}, B_{2}$ and the subsequent checks can be computed in time polynomial in the size of the input.

\section{An Application to Coordination Games}

Building on pioneering contributions by Emile Borel and John von Neumann, the first fully-fledged general characterisation of the conditions for the existence of equilibria in strategic-form games was put forward by John Nash in [42]. This provided a principled answer to the question as to how to think and act rationally in strategic situations, which also is the unique such answer if exactly one equilibrium exists. However, it is easy to construct games with multiple equilibria, which, as noted by Schelling in [46], model quite closely a number of real-world social situations. A game with multiple payoff-indistinguishable equilibria is called a pure coordination game. If equilibria are payoff-distinguishable they are called coordination games. These games put on display a situation of strategic uncertainty, where rational players are faced with the problem of selecting an equilibrium among many. Coordination games are of particular interest given that some equilibria are more rewarding than others for all players. Typically, these games embody a tension between efficiency and security (or risk-dominance), as players choosing strategies that support payoffsuperior equilibria incur in greater losses if their choice is not matched by their opponent. As a consequence, two types of coordination failure may occur: a disequilibrium outcome and coordination on a suboptimal equilibrium. Harsanyi and Selten (see [31]) formalised the tradeoff implicit in these games and stated that rational players, in the presence of common knowledge of rationality, should select the efficient (i.e., payoff-dominant) equilibrium. However, subsequent experimental studies convincingly demonstrated that Harsanyi and Selten's prediction holds true only under very special conditions, while in all remaining cases coordination failure occurs almost invariably. Building on this, [12] showed that common knowledge of the recommendation to play (one's part of) the efficient equilibrium is not conducive to coordinating on the most rewarding equilibrium. In short, the problem of identifying the rational course of action in a multiple equilibrium game remains essentially open.

In this section we suggest that possibilistic equilibria may play an interesting role in addressing the problem. To do so, we cast a paradigmatic example of coordination game - the so-called weaklink (WLG) a.k.a. minimum effort game - in the language of strategic-form games with possibilistic mixed strategies. On-time aircraft departures are prototypical examples of coordination problems of the weak-link type (see, e.g., [37]), since the airplane cannot take-off before all operations (e.g., fuelling, security checks, loading of luggage, boarding of passengers, etc.) have been completed. Other examples include relationships between different branches of a bank, the writing of a grant 
proposal involving several participants, an edited volume involving several authors, and many others $[3,4]$. Common to all aforementioned examples is the fact that the output is determined by the agent exerting the lowest level of effort (the "weak link"), and any effort above the minimum is wasted.

Here we interpret possibilistic mixed strategies as an index of commitment of a player towards their strategies. Analysing the WLG helps us putting forward a significant distinction between Choquet and Sugeno expectations to the extent that Choquet equilibria do provide us with a symmetry-breaking device which singles out the commitment to play higher-payoff (and hence higher-risk) strategies (Theorem 27). Since WLGs are typically seen as models for analysing the tradeoffs between payoff-dominant and risk-dominant equilibria, the existence of Choquet equilibria feeds back suggesting that the use of possibility distributions and Choquet expectation offers a novel approach to the refinement and selection of Nash Equilibria. In addition, it supports our interpretation of possibilistic mixed strategies as an index of commitment of a player towards their available choices.

\subsection{Weak-link Games}

A weak-link game is defined by $n$ players who must simultaneously choose a natural number $x$ in $\{1,2, \ldots, q\}$. The payoff function for the game is defined as follows:

$$
u_{i}\left(x_{1}, x_{2}, \ldots x_{n}\right)=a+b \cdot \min \left(x_{1}, x_{2}, \ldots x_{n}\right)-c \cdot\left[x_{i}-\min \left(x_{1}, x_{2}, \ldots x_{n}\right)\right]
$$

where $x_{i}$ is the number chosen by player $i$, and $a, b$ and $c$ are positive parameters. $x_{i}$ is intuitively interpreted as the "effort level chosen by player $i$ ". Provided that the payoff function is common knowledge, the game has $n$ pure Nash equilibria, corresponding to the $n$ action combinations in which all players select the same effort level. Furthermore, the Nash equilibria can be Paretoranked, with the combination

$$
\left\{x_{1}=x_{2}=\cdots=x_{n}=q\right\}
$$

being the efficient, or payoff-dominant equilibrium. Vice versa, the combination

$$
\left\{x_{1}=x_{2}=\cdots=x_{n}=1\right\}
$$

corresponds to the secure equilibrium, see [31].

In this work, we only consider WLGs with two players. We denote by $\mathbf{W}$ any weak-link game where each player $i \in\{1,2\}$ has a non-empty strategy set

$$
S=\{1, \ldots, q\}
$$

with $q \in \mathbb{N}$, and where

$$
u_{i}\left(x_{1}, x_{2}\right)=a+b \cdot \min \left(x_{1}, x_{2}\right)-c \cdot\left[x_{i}-\min \left(x_{1}, x_{2}\right)\right]
$$

is a non-negative function.

In the next subsections, we will study the Choquet and Sugeno mixed extensions of WLGs. Each player $i \in\{1,2\}$ will be assumed to have the full set $\Sigma$ of possibility distributions over their strategy set, i.e. each $\pi_{i} \in \Sigma$ is such that

$$
\pi_{i}: S \rightarrow[0,1]
$$


(notice that the set of possibilistic mixed strategies $\Sigma$ is the same for each player). A possibility distribution is interpreted as a ranking or an index specifying the commitment for a player to play a certain strategy. If the players assign the same degree of possibility towards a strategy $s \in S$, i.e. $\pi_{1}(s)=\pi_{2}(s)$, we say that the players agree on $s$. In other words, both players have the same commitment towards playing the same strategy and making the same effort.

Example 25. Let $\mathbf{W}_{7}$ be the $W L G$ with the following parameter values: $n=2, q=7, a=6$, $b=c=1$. The payoff functions of $\mathbf{W}_{7}$ are defined as follows, for each player $i$ :

$$
u_{i}\left(x_{1}, x_{2}\right)=6+\min \left(x_{1}, x_{2}\right)-\left[x_{i}-\min \left(x_{1}, x_{2}\right)\right] .
$$

For these parameters, the game presents seven Pareto-ranked Nash equilibria, with the combination

$$
x_{1}=x_{2}=7
$$

representing the payoff-dominant equilibrium and the combination

$$
x_{1}=x_{2}=1
$$

the secure equilibrium. Each player is penalised the further their choice is from the minimum in the group. The resulting payoff matrix is shown in Table 1.

\section{Player 2}

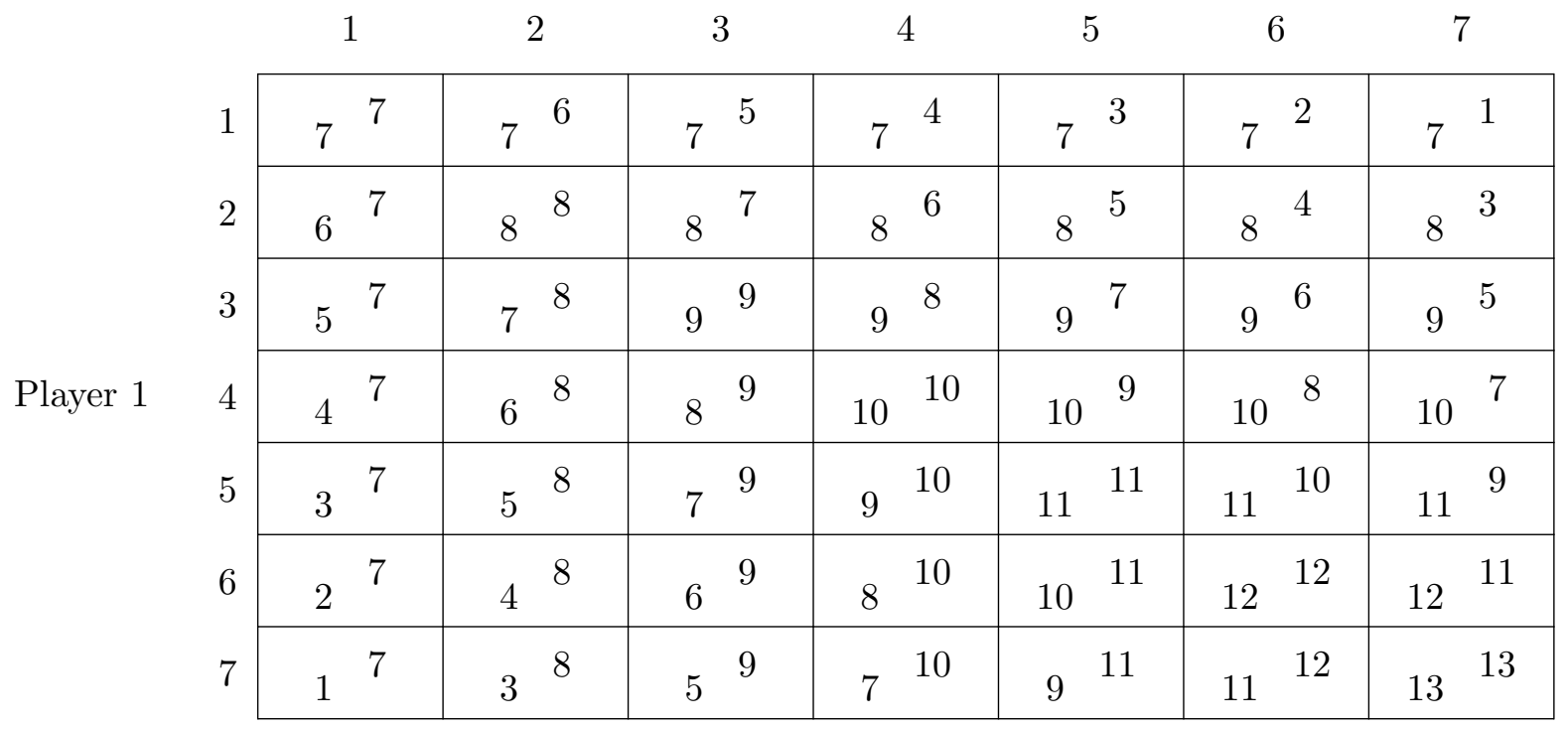

Table 1: Payoff of the 2-player weak-link game $\mathbf{W}_{7}$.

\subsection{Quantitative Approach}

To understand how to use the concept of Choquet expectation for WLGs, we explain how it is computed for the game $\mathbf{W}_{7}$. Let $\left(\pi_{1}, \pi_{2}\right) \in \Sigma \times \Sigma$ be a pair of possibilistic mixed strategies. The 
first step is to compute the joint distribution $\pi_{\times}$of the players from the marginal distributions $\pi_{1}, \pi_{2}$. Take a permutation $\alpha_{i}$ on $S \times S$ such that

$$
u_{i}\left(\left(s, s^{\prime}\right)_{\alpha_{i}(1)}\right) \leq u_{i}\left(\left(s, s^{\prime}\right)_{\alpha_{i}(2)}\right) \leq \cdots \leq u_{i}\left(\left(s, s^{\prime}\right)_{\alpha_{i}(49)}\right),
$$

and the sets $A_{\alpha_{i}(j)} \subseteq S \times S$ as

$$
A_{\alpha_{i}(j)}=\left\{\left(s, s^{\prime}\right)_{\alpha_{i}(j)},\left(s, s^{\prime}\right)_{\alpha_{i}(j+1)}, \ldots,\left(s, s^{\prime}\right)_{\alpha_{i}(49)}\right\} .
$$

For each $A_{\alpha_{i}(j)}$, compute its possibility value

$$
\Pi\left(A_{\alpha_{i}(j)}\right)=\bigvee_{\left(s, s^{\prime}\right) \in A_{\alpha_{i}(j)}}\left(\pi_{1}(s) \wedge \pi_{2}\left(s^{\prime}\right)\right) .
$$

The Choquet expectation for player $i$ is given by

$$
E_{i}^{\mathcal{C} h}\left(\pi_{1}, \pi_{2}\right)=\sum_{j=1}^{49}\left(u_{i}\left(\left(s, s^{\prime}\right)_{\alpha_{i}(j)}\right)-u_{i}\left(\left(s, s^{\prime}\right)_{\alpha_{i}(j-1)}\right)\right) \cdot \Pi\left(A_{\alpha_{i}(j)}\right) .
$$

The above definition can be significantly simplified. Let $J$ be the set of all $1 \leq j \leq 49$ such that $u_{i}\left(\left(s_{1}, s_{2}\right)_{\alpha_{1}(j)}\right)-u_{i}\left(\left(s_{1}, s_{2}\right)_{\alpha_{i}(j-1)}\right)>0$. For each $j^{\prime} \in J$

$$
u_{i}\left(\left(s, s^{\prime}\right)_{\alpha_{i}\left(j^{\prime}\right)}\right)-u_{i}\left(\left(s, s^{\prime}\right)_{\alpha_{i}\left(j^{\prime}-1\right)}\right)=1 .
$$

Then we have that

$$
E_{i}^{\mathcal{C h}}\left(\pi_{1}, \pi_{2}\right)=\sum_{j^{\prime} \in J} \Pi\left(A_{\alpha_{i}\left(j^{\prime}\right)}\right) .
$$

From Corollary 14, we know a CE for WLGs always exists and it coincides with the trivial equilibrium in which both player 1 and player 2 assign maximum value to each single strategy. This situation coincides with the case where none of the players actually cares about the outcome, i.e. they are indifferent on which strategy to play. This is not the only equilibrium and, in fact, as we prove below, every situation in which both players agree on all strategies, i.e. they have the same commitment to play each strategy, is also an equilibrium. Full agreement though is not required for the existence of an equilibrium, as shown in the following example.

Example 26. Consider the weak-link game $\mathbf{W}_{7}$ and the following pair of possibilistic mixed strategies:

$$
\pi_{1}=(0,0,0.5,0.5,1,0.3,0.5) \quad \pi_{2}=(1,1,0.5,0.5,1,0.4,0.5) .
$$

It is clear that the players' distributions are quite different in the pair $\left(\pi_{1}, \pi_{2}\right)$. To see that $\left(\pi_{1}, \pi_{2}\right)$ is a CE, we check whether condition (2) of Theorem 13 is satisfied. Table 2 and Table 3 give a visual representation of this process. For each $1 \leq j \leq 49$ such that

$$
u_{i}\left(\left(s_{1}, s_{2}\right)_{\alpha_{1}(j)}\right)-u_{i}\left(\left(s_{1}, s_{2}\right)_{\alpha_{i}(j-1)}\right)>0:
$$

- The cells highlighted in light grey correspond to the strategy combinations $\left(s, s^{\prime}\right) \in A_{\alpha_{i}(j)}$. 

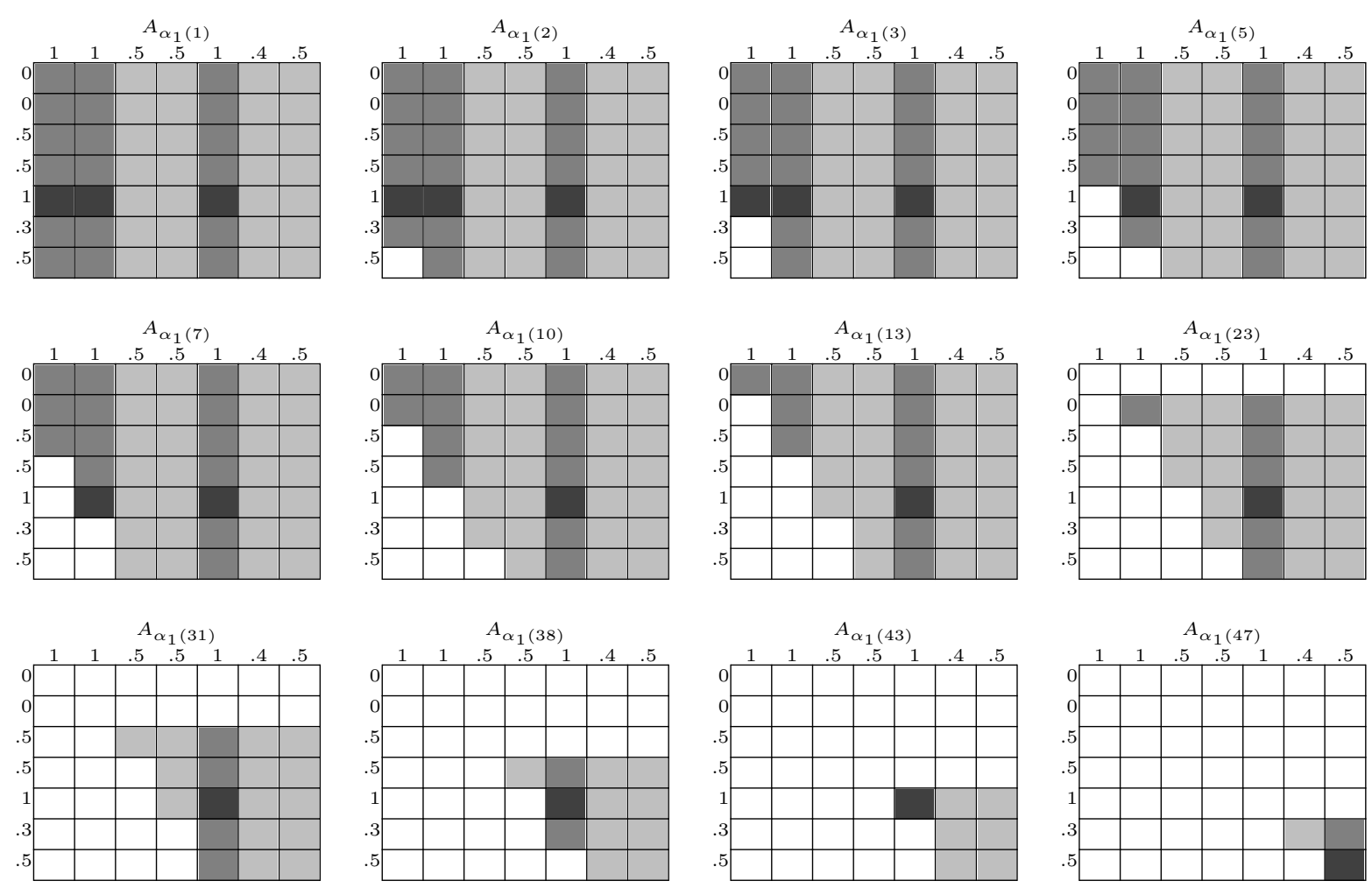

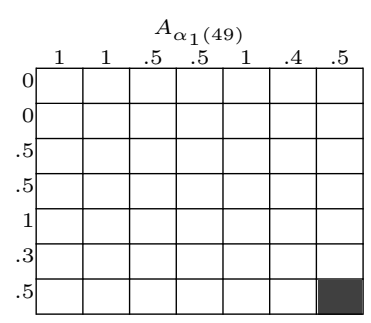

Table 2: $A_{\alpha_{1}(j)}$ sets in the weak-link game $\mathbf{W}_{7}$ (Example 26).

- The white cells correspond to the strategy combinations $\left(s, s^{\prime}\right) \notin A_{\alpha_{i}(j)}$.

- The cells highlighted in grey correspond to those strategy combinations that belong to $A_{\alpha_{i}(j)}^{\max }$ (see Theorem 13).

- The cells highlighted in dark grey correspond to the strategy profiles $\left(s, s^{\prime}\right) \in A_{\alpha_{i}(j)}^{\max }$ for which

$$
\pi_{i}\left(s_{i}\right) \geq \pi_{-i}\left(s_{-i}\right) .
$$

Table 2 and Table 3 show that Condition (2) of Theorem 13 is satisfied and so $\left(\pi_{1}, \pi_{2}\right)$ is a CE.

The above example shows that for a WLG disagreement in terms of having a different commitment over the possible choices does not negate a CE. In this case, while the players heavily disagree on the first strategies, they show a much higher level of agreement over strategies to play higher numbers (i.e. over strategies that have more effort in the WLG). This is a key point for the 

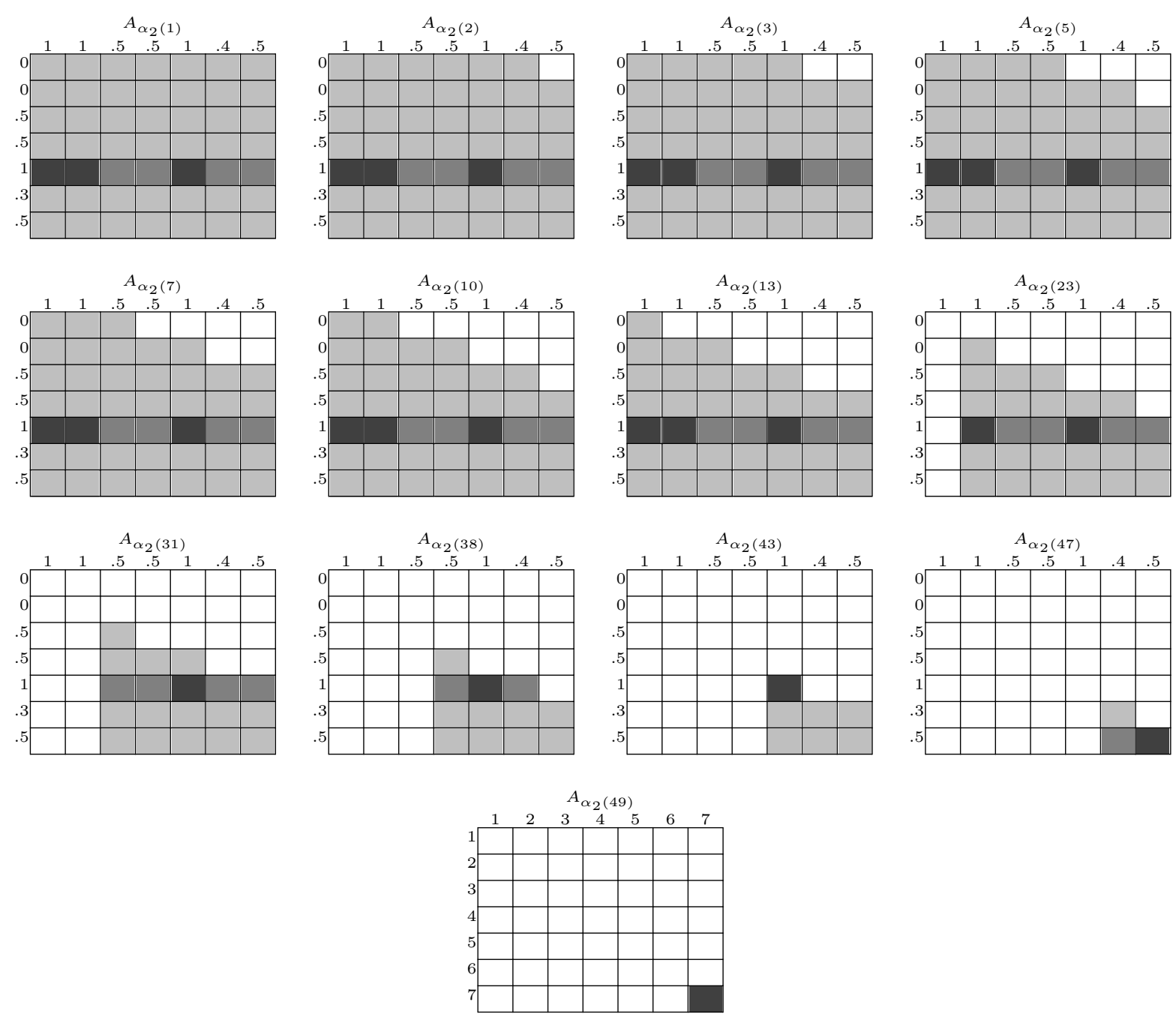

Table 3: $A_{\alpha_{2}(j)}$ sets in the weak-link game $\mathbf{W}_{7}$ (Example 26). 
existence of CEs in WLGs, and is a consequence of a more general characterisation that we give here below. This characterisation is specific to WLGs and simplifies the one given in Theorem 13.

Theorem 27. Let $\mathbf{W}$ be any weak-link game, let $\mathfrak{W}$ be its Choquet mixed extension and let $\left(\pi_{1}, \pi_{2}\right) \in \Sigma \times \Sigma$. The following statements are equivalent:

1. $\left(\pi_{1}, \pi_{2}\right)$ is a Choquet equilibrium for $\mathbf{W}$;

2. For all $s \in S$, if $\pi_{1}(s) \neq \pi_{2}(s)$, then $s \neq q$ and there exists $s^{\prime} \in S$ such that $s^{\prime}>s$ and

$$
\pi_{1}\left(s^{\prime}\right)=\pi_{2}\left(s^{\prime}\right) \geq \pi_{1}(s) \vee \pi_{2}(s) .
$$

Proof. (1) $\Rightarrow(2)$ : Suppose (2) does not hold. Let $Y \subseteq S$ be the set of strategies for which condition

(2) fails and let $s$ be its greatest element. We have that either $s=q$ or for all $s^{\prime}>s$

(a) $\pi_{1}\left(s^{\prime}\right) \neq \pi_{2}\left(s^{\prime}\right)$ and $s^{\prime}$ satisfies condition (2), or

(b) $\pi_{1}\left(s^{\prime}\right)=\pi_{2}\left(s^{\prime}\right)<\pi_{1}(s) \vee \pi_{2}(s)$.

Let us assume, without any loss of generality, that

$$
\pi_{1}(s)<\pi_{2}(s) .
$$

If $s=q$, then $\left(\pi_{1}, \pi_{2}\right)$ is not a CE. In fact $A_{\alpha_{1}\left(q^{2}\right)}$ contains only the element $(q, q)$ and, since

$$
\pi_{1}(q)<\pi_{2}(q)
$$

condition (2) of Theorem 13 fails.

Assume then that $s<q$. For all $s^{\prime}>s$ we have that

$$
\pi_{2}\left(s^{\prime}\right)<\pi_{2}(s) .
$$

In fact, if $s^{\prime}$ satisfies $(b)$ then

$$
\pi_{1}\left(s^{\prime}\right)=\pi_{2}\left(s^{\prime}\right)<\pi_{1}(s) \vee \pi_{2}(s)=\pi_{2}(s) .
$$

If $s^{\prime}$ satisfies $(a)$, then it must be the case that

$$
\pi_{2}\left(s^{\prime}\right)<\pi_{1}(s) \vee \pi_{2}(s)=\pi_{2}(s),
$$

since condition (2) fails for $s$.

Now, let $A_{\alpha_{1}(j)}$ be the smallest set containing all strategy combinations $\left(s^{\prime \prime}, s^{\prime \prime \prime}\right)$ such that $u_{1}(s, s) \leq u_{1}\left(s^{\prime \prime}, s^{\prime \prime \prime}\right) . A_{\alpha_{1}(j)}$ contains all pairs $\left(s^{\prime}, s^{\prime}\right)$ with $s^{\prime}>s$, and, since we have established that

$$
\pi_{2}\left(s^{\prime}\right)<\pi_{2}(s)
$$

it is easy to see that

$$
A_{\alpha_{1}(j)}^{\max }=\{(s, s)\} .
$$

We have assumed that

$$
\pi_{1}(s)<\pi_{2}(s),
$$

consequently, condition (2) of Theorem 13 fails and $\left(\pi_{1}, \pi_{2}\right)$ is not a CE. 
$(2) \Rightarrow(1)$ : Suppose $(1)$ does not hold, i.e. $\left(\pi_{1}, \pi_{2}\right)$ is not a CE. Without any loss of generality, by Theorem 13, we can assume that for player 1 , there exists $1 \leq j \leq q^{2}$ such that

$$
u_{1}\left(\left(s_{1}, s_{2}\right)_{\alpha_{1}(j)}\right)-u_{1}\left(\left(s_{1}, s_{2}\right)_{\alpha_{1}(j-1)}\right)>0
$$

so that for all $\left(s, s^{\prime}\right) \in A_{\alpha_{1}(j)}^{\max }$,

$$
\pi_{1}(s)<\pi_{2}\left(s^{\prime}\right)
$$

Now, notice that for all $\left(s, s^{\prime}\right) \in A_{\alpha_{1}(j)}^{\max }$, also $\left(s^{\prime}, s^{\prime}\right) \in A_{\alpha_{1}(j)}$. In fact, it is easy to check that, in general,

$$
u_{1}\left(s, s^{\prime}\right) \leq u_{1}\left(s^{\prime}, s^{\prime}\right)
$$

If $s \leq s^{\prime}$

$$
\begin{gathered}
u_{1}\left(s, s^{\prime}\right)=a+b \cdot \min \left(s, s^{\prime}\right)-c \cdot\left(s-\min \left(s, s^{\prime}\right)\right)=a+b \cdot s, \\
u_{1}\left(s^{\prime}, s^{\prime}\right)=a+b \cdot \min \left(s^{\prime}, s^{\prime}\right)-c \cdot\left(s^{\prime}-\min \left(s^{\prime}, s^{\prime}\right)\right)=a+b \cdot s^{\prime},
\end{gathered}
$$

and so

$$
u_{1}\left(s, s^{\prime}\right)=a+b \cdot s \leq a+b \cdot s^{\prime}=u_{1}\left(s^{\prime}, s^{\prime}\right)
$$

If $s \geq s^{\prime}$

$$
\begin{gathered}
u_{1}\left(s, s^{\prime}\right)=a+b \cdot \min \left(s, s^{\prime}\right)-c \cdot\left(s-\min \left(s, s^{\prime}\right)\right)=a+b \cdot s^{\prime}-c \cdot\left(s-s^{\prime}\right), \\
u_{1}\left(s^{\prime}, s^{\prime}\right)=a+b \cdot \min \left(s^{\prime}, s^{\prime}\right)-c \cdot\left(s^{\prime}-\min \left(s^{\prime}, s^{\prime}\right)\right)=a+b \cdot s^{\prime},
\end{gathered}
$$

and so

$$
u_{1}\left(s, s^{\prime}\right)=a+b \cdot s^{\prime}-c \cdot\left(s-s^{\prime}\right) \leq a+b \cdot s^{\prime}=u_{1}\left(s^{\prime}, s^{\prime}\right) .
$$

This shows that $\left(s^{\prime}, s^{\prime}\right) \in A_{\alpha_{1}(j)}$ and consequently $\left(s^{\prime}, s^{\prime}\right) \in A_{\alpha_{1}(j)}^{\max }$, which means that

$$
\pi_{1}\left(s^{\prime}\right)<\pi_{2}\left(s^{\prime}\right) .
$$

If $s^{\prime}=q$, we have reached our conclusion. Otherwise, suppose that $s^{\prime}<q$ and for some $s^{\prime \prime}>s^{\prime}$,

$$
\pi_{1}\left(s^{\prime \prime}\right)=\pi_{2}\left(s^{\prime \prime}\right) \geq \pi_{1}\left(s^{\prime}\right) \vee \pi_{2}\left(s^{\prime}\right) .
$$

It is clear that $\left(s^{\prime \prime}, s^{\prime \prime}\right) \in A_{\alpha_{1}(j)}$ and also $\left(s^{\prime \prime}, s^{\prime \prime}\right) \in A_{\alpha_{1}(j)}^{\max }$. This contradicts our original assumption, so it must be the case that (2) does not hold.

This concludes the proof of the theorem.

In spite of the technicalities, the above result gives us a fairly clear picture of whether two possibility distributions form a Choquet equilibrium. While this always trivially happens when players fully agree in their distributions, disagreement is clearly tolerated. That is the case whenever disagreement in playing a certain strategy is superseded by fully agreeing to playing a strategy with a higher effort. In other words: for an equilibrium to exist, any time two players have a different possibility to play a certain strategy, they must both have a higher and the same possibility to play a strategy that requires a greater effort than the one they disagreed upon.

In this sense, Choquet equilibria can be seen to provide an interesting refinement of Nash equilibria. To see this, recall that the coordination games, of which the WLG provides a special case, raise the question of selecting among payoff-distinguishable equilibria which nonetheless are all Nash equilibria. So, selecting from this set effectively means achieving two goals. First, the 
symmetry among equilibria must be broken. Second, it must be broken in such a way that the most desirable equilibria become salient. For the WLG, higher payoff equilibria clearly count as such. The significance of Theorem 27 can then be analysed again from this perspective. We have two cases to consider. Suppose that player 1 and player 2 agree fully on their possibility distributions on $S$ for game W. Then possibilistic mixed strategies do not allow us to make any distinction among the set of Nash equilibria. This however should not be surprising, for any method for selecting among Nash equilibria must break the symmetry of the coordination game, a task that certainly cannot be accomplished by a pair of identical possibility distributions. So, symmetry must be broken by some form of disagreement between $\pi_{1}$ and $\pi_{2}$, which brings us to the second, more interesting, case. What our analysis of Choquet equilibria in the WLG brings forward is then that such a disagreement makes higher payoff strategies more salient. For disagreement on lower payoff strategies, in a Choquet Equilibrium, is compensated by agreement on payoff superior ones. This is precisely the kind of refinement of Nash equilibrium desirable for the WLG.

\subsection{Qualitative Approach}

As mentioned in Remark 18, whenever we deal with Sugeno expected utility, we assume strategicform games to have utility functions $u_{i}, i \in\{1,2\}$, such that

$$
\operatorname{ran}\left(u_{i}\right)=\left\{0, a_{1}, \ldots, a_{m_{i}-2}, 1\right\} \subset[0,1]
$$

so that their range belong to the same scale as the possibility distributions. In the weak-link game, each utility function is of the form

$$
u_{i}:\{1, \ldots, 7\} \times\{1, \ldots, 7\} \rightarrow\{1, \ldots, 13\},
$$

so, in order to use Sugeno expected utility, we have two options:

1. We rescale the range of $u_{i}$ and define an order-preserving bijection

$$
h:\{1, \ldots, 13\} \rightarrow\left\{0, \frac{1}{12}, \ldots, \frac{11}{12}, 1\right\}
$$

to obtain new utility functions

$$
u_{i}^{\prime}=h\left(u_{i}\right)
$$

and define a new game that is strategically equivalent to the WLG.

2. We rescale the range of the possibility distributions $\pi_{i}$ so that, for each player $i$,

$$
\pi_{i}:\{1,2, \ldots, 7\} \rightarrow[1,13] .
$$

In this section, we follow the second approach. Notice that the setting and the results of Section 3.2 easily hold.

Recall that the expected utility for each player $i$ is given by

$$
E_{i}^{\mathcal{S}}\left(\pi_{1}, \pi_{2}\right)=\bigvee_{\left(s_{1}, s_{2}\right) \in S_{1} \times S_{2}}\left(u_{i}\left(\left(s_{1}, s_{2}\right)\right) \wedge\left(\pi_{1}\left(s_{1}\right) \wedge \pi_{2}\left(s_{2}\right)\right)\right)
$$

From Corollary 23, we know that a SE always exists and coincides with the case where both distributions assign maximum value to all the strategies. This is not the only equilibrium and, in 
fact, there are many, such as those that correspond to situations in which both players agree on all strategies, meaning that they have the same commitment toward playing the same strategies.

Similar to the case of Choquet expectation, equilibria exist also in the presence of disagreement. Consider first situations with extremal distributions (i.e. distributions assigning to each strategy either the maximum or the minimum) for both players. A SE can exist even when players disagree on their extremal distributions. For instance, with the distributions

$$
\pi_{1}=(13,1,1,13,13,1,1) \quad \pi_{2}=(1,13,13,1,13,1,1),
$$

player 1 and player 2 disagree for strategies from 1 to 4 , but agree on the remaining strategies. We can use Theorem 22 to see that $\left(\pi_{1}, \pi_{2}\right)$ is a SE. In fact

$$
B_{1}=\{(5,5)\} \quad \text { and } \quad \pi_{1}(5)=13 \geq\left(\pi_{2}(5) \wedge u_{1}(5,5)\right)=(13 \wedge 11)=11
$$

and

$$
B_{2}=\{(5,5)\} \quad \text { and } \quad \pi_{2}(5)=13 \geq\left(\pi_{1}(5) \wedge u_{2}(5,5)\right)=(13 \wedge 11)=11 .
$$

In this case both players assign the maximum value to different strategies but agree on a strategy for which the related effort is greater than the effort for those strategies for which they have a different commitment.

The case of non-extremal distributions is similar. While full, agreement always guarantees an equilibrium, disagreement does not necessarily negate the SE. Take for instance, the distributions

$$
\pi_{1}^{\prime}=(13,7,13,5,12,6,3) \quad \pi_{2}^{\prime}=(3,13,1,1,12,2,2) .
$$

Both players heavily disagree over many strategies but agree to a very high degree on strategy 5 . The fact that $\left(\pi_{1}^{\prime}, \pi_{2}^{\prime}\right)$ is a SE is shown by the following:

$$
B_{1}=\{(5,5)\} \quad \text { and } \quad \pi_{1}^{\prime}(5)=12 \geq\left(\pi_{2}^{\prime}(5) \wedge u_{1}(5,5)\right)=(12 \wedge 11)=11 ;
$$

and

$$
B_{2}=\{(5,5)\} \quad \text { and } \quad \pi_{2}^{\prime}(5)=12 \geq\left(\pi_{1}^{\prime}(5) \wedge u_{2}(5,5)\right)=(12 \wedge 11)=11 .
$$

As another example, consider the distributions

$$
\pi_{1}^{\prime \prime}=(13,1,6,1,6,1,6) \quad \pi_{2}^{\prime \prime}=(13,6,1,6,1,6,1) .
$$

Both players have the same and highest commitment to play 1 and heavily disagree on all the other strategies. Still, the pair $\left(\pi_{1}^{\prime \prime}, \pi_{2}^{\prime \prime}\right)$ is a Sugeno equilibrium. In fact:

$$
B_{1}=\{(1,1)\} \quad \text { and } \quad \pi_{1}^{\prime \prime}(1)=13 \geq\left(\pi_{2}^{\prime \prime}(1) \wedge u_{1}(1,1)\right)=(13 \wedge 7)=7 ;
$$

and

$$
B_{2}=\{(1,1)\} \quad \text { and } \quad \pi_{2}^{\prime \prime}(1)=13 \geq\left(\pi_{1}^{\prime \prime}(1) \wedge u_{2}(1,1)\right)=(13 \wedge 7)=7 .
$$

The above examples of the WLG with with Sugeno expectation suggest that agreement among players with respect to their commitment to playing certain strategies is always better but disagreement is not detrimental. The situation shares some similarities with Choquet expectation, but there are some crucial differences. The first one is in the role of the utility function and how the expected value is computed. In the case of Choquet expectation, utility functions play no 
role in the existence of equilibria. Theorem 27 clearly shows that comparing the values of the possibility distributions is the only thing that matters. In Sugeno expectation, however, the values of the utility functions play a fundamental role. This is evident from the way the Sugeno integral is defined and also from the characterisation given in Theorem 22 and is due to its essentially qualitative nature. In a Choquet integral the value of a possibility measure is multiplied by the difference between values of the utility function. The possibilistic value can then be seen as a sort of modifier or weight. In a Sugeno integral the values of a possibility measure (distribution) and of the utility function are combined with the minimum function. The result of this combination is either one value or the other. The possibilistic value does not simply act as a modifier or a weight but is treated as an entity equal to the the utility and has to be compared to it. So, when dealing with Sugeno equilibria for the WLG, we cannot take into account only the possibilistic mixed strategies and the players' commitment. Their utility functions will always play a role.

Another clear difference between Sugeno and Choquet expectation emerges in situation of disagreement. Disagreement between possibilistic mixed strategies in a Choquet equilibrium is resolved by the players agreeing to play a same strategy with higher effort with a higher commitment than the strategy they disagreed upon. As proven in Theorem 27, agreement on strategies with higher effort is unavoidable in a CE. For Sugeno expectation this kind of agreement is not necessary for the equilibrium to exist. In the distributions

$$
\pi_{1}^{\prime}=(13,7,13,5,12,6,3) \quad \text { and } \quad \pi_{2}^{\prime}=(3,13,1,1,12,2,2),
$$

players agree on 5, but clearly disagree on both 6 and 7 . Even more notable is the case of distributions

$$
\pi_{1}^{\prime \prime}=(13,1,6,1,6,1,6) \quad \text { and } \quad \pi_{2}^{\prime \prime}=(13,6,1,6,1,6,1),
$$

where players fully agree on 1 , but substantially disagree on all the strategies with higher effort. This shows that in situations of disagreement, Sugeno expectation is not conducive to the commitment to higher payoff strategies in the same way Choquet expectation is.

For these reasons we believe that Sugeno expectation cannot play a prominent role in the selection of multiple Nash equilibria when applied to WLGs.

\section{Conclusions and further work}

Randomisation was introduced in the analysis of strategic-form games as a tool to solve the existence of equilibria. It was originally understood as probabilistic randomisation, since, in the early development of game theory (culminated in the 1950s), there was hardly any choice other than probability theory in the formal modelling of uncertainty. Over the past few decades, a variety of alternative approaches to uncertainty has been investigated in the wider field of uncertain reasoning (see [29, 47]) and it is only natural to ask which non-probabilistic measures of uncertainty can be used consistently for randomising purposes and how this may contribute to the analysis of strategic interaction.

We devoted the initial part of this paper to trying to answer this question for the possibilistic setting. We extended strategic-form games with a concept of randomisation formally encoded through possibilistic mixed strategies. We revisited the usual notion of equilibrium in this framework and studied its game-theoretic and computational properties. In particular, we investigated two distinct instantiations of the notion of possibilistic equilibria: one based on the Choquet integral and one based on the Sugeno integral. Our main results, Theorem 13 and Theorem 22 fully 
identify the conditions under which a pair of possibilistic mixed strategies forms a Choquet or a Sugeno equilibrium, respectively.

The second part of the paper is devoted to exploring the impact of possibilistic randomisation in the formal analysis of games. In Section 4, we applied our approach to the problem of strategic uncertainty and equilibrium selection in weak-link games and put forward an interpretation of possibility distributions in terms of players' commitment. This analysis, which is carried out through representative examples, allowed us to reach two conclusions. First, the notion of possibilistic expectation can provide a formal way of distinguishing appropriately among the multiple Nash equilibria of a weak-link game. This clearly shows the potential of possibilistic equilibria in the analysis of coordination games. And it does so appropriately because it delivers a recommendation which is intuitively rational in the context of the game namely, team-up for a better collective outcome! Second, we observe that this improvement over the classical setting can be obtained only with Choquet equilibria. This provides an operational way of distinguishing two notions of possibilistic equilibrium which would otherwise be equally consistent from the purely mathematical point of view. This we regard as a contribution to the interpretation of possibility theory in the wider field of uncertain reasoning.

Our initial results into possibilistic randomisation are indeed encouraging both from the mathematical point of view and from the point of view of its applicability in game theory. Much is still to be done in this latter direction. First we will aim at investigating in full generality, rather than with representative examples, the capability of Choquet equilibria to provide a principled solution to the vexed question of selecting among multiple Nash Equilibria. This will involve testing this framework against general coordination games, including pure coordination ones, and comparing our findings with the results obtained in the field of global games (see [5, 40]). In a nutshell, the framework of global games leads to one principled way of selecting uniquely among multiple Nash equilibria in coordination games. This refinement arises, quite surprisingly, by dispensing with the assumption of "full" common knowledge among players about the payoff structure, which is obtained by introducing a small amount of "probabilistic noise" to the description of the strategies. It will then be natural to ask whether "possibilistic noise" can be defined in a way that it can be conducive to the selection of a unique Nash equilibrium in pure coordination games.

The second major item for future research on possibilistic randomisation will be concerned with exploring the viability of extending our commitment interpretation to all strategic-form games. While in Section 4, we explicitly interpret possibilistic mixed strategies as an index of players' commitment and obtain a new and interesting analysis of weak-link games, in the rest of the paper we do not assume any interpretation of what possibilistic randomisation is: we were, in fact, interested in exploring the general properties of our models and obtaining mathematical results independent of any interpretation. Still, as briefly recalled in the introductory section, delving deeper in the behavioural interpretation of possibilistic randomisation is a natural next step.

Indeed the non-probabilistic analysis of equilibrium in beliefs is a question which has recently attracted significant attention in economic theory. Recall that the notion of (probabilistic) randomisation plays a role in two distinct concepts of interest in game theory. The first is equilibrium in actions, the second is equilibrium in beliefs. Although the seminal work of Harsanyi [30] connects the two, they lend themselves to distinct levels of analysis. Our present interest lied with the exploration of the mathematical consequences of assuming that indifference between pure actions is modelled by possibilistic randomisation. The results we obtained by asking this question do not bear directly, and hence are not comparable with those of the rich economic literature 
which investigates how equilibria in beliefs can be generalised to non-probabilistic measures of uncertainty. In particular, Eichberger and Kelsey studied in [23] games where players' beliefs about their opponents' behaviour are modelled as non-additive probabilities. In a similar fashion, Marinacci introduced in [38] ambiguous games, an extension of strategic-form games that allows the presence of vagueness in players' beliefs over the opponents' choice of strategies. Both in Eichberger-Kelsey's and Marinacci's approach, beliefs are formalised by convex capacities (therefore excluding possibility measures, which are not convex [27]) and expectation is defined with the Choquet integral [7]. Future investigations on the extension of the results of the present paper to providing a behavioural foundation to possibilistic mixed strategies will certainly have to start from this background in conjunction with the results obtained in [44].

Further research will be needed to understand whether this approach can be fully and meaningfully generalised in order to offer a coherent and satisfactory behavioural interpretation of possibilistic randomisation.

\section{Acknowledgements}

We would like to thank the two anonymous reviewers who helped us improve this paper significantly through unrestrained criticisms. We would also like to thank the Editors for their patience and competence.

[1] N. Ben Amor, H. Fargier, R. Sabbadin. Equilibria in Ordinal Games: A Framework based on Possibility Theory. In Proceedings of the Twenty-Sixth International Joint Conference on Artificial Intelligence (JCAI-17), 105-111, 2017

[2] G. Bonanno. Game Theory. 2nd ed. 2018. http://faculty.econ.ucdavis.edu/faculty/bonanno/.

[3] C. Camerer, M. Knez. Coordination, Organizational Boundaries and Fads in Business Practice, Industrial and Corporate Change, 5: 89-112, 1996.

[4] C. Camerer, M. Knez. Coordination in Organizations: A Game-theoretic Perspective. In Z. Shapira (Ed.), Organizational decision making, Cambridge: Cambridge Series on Judgment and Decision Making, 158-188, 1997.

[5] H. Carlsson, E. van Damme. Global games and equilibrium selection. Econometrica, 61: 989-1018, 1993.

[6] A. Chateauneuf, M. Grabisch, A. Rico. Modeling attitudes toward uncertainty through the use of the Sugeno integral. Journal of Mathematical Economics, 44: 1084-1099, 2008

[7] G. Choquet. Theory of capacities. Annales de l'Institut Fourier, 5:131-295, 1953.

[8] S. De Clercq, S. Schockaert, A. Nowé, and M. De Cock. Modelling incomplete information in Boolean games using possibilistic logic. International Journal of Approximate Reasoning, 93: 1-23, 2018.

[9] G. De Cooman. Possibility theory Part I: Measure- and integral-theoretics groundwork; Part II: Conditional possibility; Part III: Possibilistic independence. International Journal of General Systems, 25(4): 291-371, 1997.

[10] G. De Cooman. Integration and conditioning in numerical possibility theory. Annals of Mathematics and AI, 32: 87-123, 2001.

[11] D. Denneberg. Non-Additive Measure and Integral. Kluwer, The Netherlands, 1994.

[12] G. Devetag, H. Hosni, G. Sillari. You Better Play 7 : Mutual versus Common Knowledge of Advice in a Weak-link Experiment. Synthese, 190(8): 1351-1381, 2013.

[13] D. Dubois. Possibility theory and statistical reasoning. Computational Statistics and Data Analysis, 51(1), 47-69, 2006.

[14] D. Dubois, H. Fargier. Making Discrete Sugeno Integrals More Discriminant. International Journal of Approximate Reasoning. 50(6): 880-898, 2009.

[15] D. Dubois, L. Godo, H. Prade, and A. Zapico. On the possibilistic decision model: from decision under uncertainty to case-based decision. International Journal of Uncertainty, Fuzziness and Knowledge-based Systems 7(6): 631-670, 1999.

[16] D. Dubois, J.L. Marichal, H. Prade, M. Roubens, and R. Sabbadin. The use of the discrete Sugeno integral in decision-making: A survey. International Journal of Uncertainty, Fuzziness and Knowledge-Based Systems, 9(5): 236-256, 2001. 
[17] D. Dubois, H. Prade. Weighted Minimum and Maximum Operations in Fuzzy Set Theory. Information Sciences, 39(2): 205-210, 1986.

[18] D. Dubois, H. Prade. Possibility theory. Plenum Press, New York, french edition, 1988. An approach to computerized processing of uncertainty. With the collaboration of Henri Farreny, Roger Martin-Clouaire and Claudette Testemale, Translated by E. F. Harding, With a foreword by Lotfi A. Zadeh.

[19] D. Dubois, H. Prade. Possibility Theory as a basis for Qualitative Decision Theory. Proceedings of the 14th International Joint Conference on Artificial Intelligence (IJCAI95), Montreal, 1924-1930, 1995.

[20] D. Dubois, H. Prade. Possibility Theory: Qualitative and Quantitative Aspects. In: Smets P. (eds), Quantified Representation of Uncertainty and Imprecision. Handbook of Defeasible Reasoning and Uncertainty Management Systems, Vol 1: 169-226. Springer, Dordrecht, 1998.

[21] D. Dubois, H. Prade. Possibilistic Logic: An Overview. In D. M. Gabbay and J. Woods (Eds.), Handbook of the History of Logic, Vol. 9, Computational Logic, Elsevier, 283-341, 2014.

[22] D. Dubois, H. Prade, R. Sabbadin Decision-theoretic foundation of qualitative possibility theory. European Journal of Operations Research, 128: 478-495, 2001.

[23] J. Eichberger, D. Kelsey. Non-additive beliefs and strategic equilibria. Games and Economic Behavior, 30: $183-215,2000$.

[24] T. Flaminio, L. Godo, H. Hosni. Coherence in the aggregate: A betting method for belief functions on manyvalued events. International Journal of Approximate Reasoning, 58: 71-86, 2015.

[25] P. Harrenstein, W. van der Hoek, J.-J.Ch Meyer and C. Witteveen C. Boolean games. In J. van Benthem, editor, Proceeding of the Eighth Conference on Theoretical Aspects of Rationality and Knowledge (TARK VIII), Siena, Italy, 287-298, 2001.

[26] M. Grabisch, T. Murofushi, M. Sugeno. Fuzzy Measure of Fuzzy Events Defined by Fuzzy Integrals. Fuzzy Sets and Systems, 50: 293-313, 1992.

[27] M. Grabisch. Set Functions, Games and Capacities in Decision Making. Springer, 2016.

[28] M. Grabisch, C. Labreuche. A decade of application of the Choquet and Sugeno integrals in multi-criteria decision aid. 4OR, 6(1): 1-44, 2008.

[29] J.Y. Halpern. Reasoning about Uncertainty. The MIT Press, Cambridge Massachusetts, 2003.

[30] J. C. Harsanyi. Games with Randomly Distributed Payoffs: A New Rationale for Mixed-Strategy Equilibrium Points. International Journal of Game Theory, 2(1):1-23, 1973.

[31] J. C. Harsanyi, R. Selten. A General Theory of Equilibrium Selection in Games. MIT Press, 1988.

[32] H. Hosni, E. Marchioni. Some Notes on Ordinal Strategic Interaction with Possibilistic Expectation. (Extended Abstract) In $34^{\text {th }}$ Linz Seminar on Fuzzy Set Theory. Linz, Austria, 2013.

[33] H. Hosni, E. Marchioni. Possibilistic Mixed-Strategies in the Selection of Multiple Nash Equilibria. (Extended Abstract). " 7th Workshop on Decision, Games 83 Logic, Stockholm, Sweden, 2013.

[34] H. Hosni, E. Marchioni. Possibilistic expectation in the selection of multiple Nash equilibria. 11th Conference on Logic and the Foundations of Game and Decision Theory July 27-30, 2014, University of Bergen, Norway

[35] R.D. Luce and H. Raiffa. Games and Decisions. Wiley, 1957.

[36] E.P. Klement, R. Mesiar, E. Pap. Triangular norms, Kluwer Academic Publishers, Dordrecht, 2000.

[37] M. Knez, D. Simester. Firm-wide incentives and mutual monitoring at Continental Airlines. Journal of Labor Economics, 19: 743-772, 2001.

[38] M. Marinacci. Ambiguous games. Games and Economic Behavior, 31: 191-219, 2000.

[39] M. Maschler, E. Solan, S. Zamir Game Theory. Cambridge University Press, 2013.

[40] S. Morris, H. Shin. Unique Equilibrium in a Model of Self-Fulfilling Currency Attacks. The American Economic Review, 88(3): 587-597. 2007

[41] R.B. Myerson. Nash Equilibrium and the History of Economic Theory. Journal of Economic Literature, 1082:1067-1082, 1999.

[42] J. Nash. Non-cooperative games. Annals of Mathematics, 54(2): 286-295, 1951.

[43] M. Osborne, A. Rubinstein. A Course in Game Theory MIT Press, 1994.

[44] T. Radul. Equilibrium under uncertainty with Sugeno payoff. Fuzzy Sets and Systems, 349(15): 64-70, 2018.

[45] E. Raufaste, R. da Silva Neves, C. Mariné. Testing the descriptive validity of possibility theory in human judgments of uncertainty. Artificial Intelligence, 148: 197-218, 2003.

[46] T. Schelling. The Strategy of Conflict (second edition). Harvard University Press, Cambridge, MA, 1980.

[47] P. Smets. Numerical representation of uncertainty. In: Handbook of Defeasible Reasoning and Uncertainty Management Systems, Dubois D. and Prade H. (Vol. Eds.), Kluwer, Doordrecht, 65-309, 1998.

[48] M. Sugeno. Theory of Fuzzy Integrals and its Applications. PhD thesis, Tokyo Institute of Technology, Tokio, Japan, 1974. 
[49] J.B. Van Huyck, R.O. Beil, A. Gillette and R.C. Battalio. Tacit Coordination Games, Strategic Uncertainty, and Coordination Failure. The American Economic Review, 80: 234-248, 1990.

[50] L.A. Zadeh. Fuzzy sets as a basis for a theory of possibility. Fuzzy Sets and Systems, 1: 3-28, 1978. 